\title{
Factores que influyen en implementación de prácticas para el alineamiento estratégico de $\mathrm{TI}$ en el sector público
}

\section{Factor that influence in implementation of practices for IT alignment strategic in the public sector}

\author{
Juan Díaz ${ }^{1, a}$, David Mauricio ${ }^{1, b}$ \\ ${ }^{1}$ Universidad Nacional Mayor de San Marcos. Lima, Perú. AI Group. \\ a Email: juankener@gmail.com \\ bEmail: dmauicios@unmsm.edu.pe
}

\begin{abstract}
Resumen
El bajo nivel de alineamiento estratégico de TI que existe en muchas entidades del sector público ocasiona que estas organizaciones no alcancen un alto desempeño, a pesar de las implementaciones de prácticas y de la gestión dadas por el gobierno de TI. Por esta razón, se han realizado estudios de los factores que influyen en la implementación de gobierno de TI y sus áreas, entre las que se destaca el alineamiento estratégico de TI. Sin embargo, aún no se ha identificado los factores que influyen en cada una de las prácticas que conducen al alineamiento estratégico, lo cual es imprescindible conocer, ya que cada práctica conforma la estrategia de $\mathrm{TI}$ de la organización y ello permitirá tomar acciones para mitigar los factores negativos o potenciar los positivos y, como consecuencia, obtener un buen alineamiento estratégico. En este trabajo se ha identificado 16 factores de implementación, 10 prácticas para el alineamiento estratégico de $\mathrm{Tl}$, en el contexto del sector público, y 48 relaciones de influencia (factor $\rightarrow$ práctica). Un estudio empírico sobre 50 ejecutivos del sector público valida 41 de las 48 relaciones planteadas con calificación entre "Alta" y "Muy Alta”, al 95 \% de confianza mediante la prueba de t-Student.
\end{abstract}

Palabras clave: Alineamiento estratégico de TI; gobierno de TI; prácticas; factores; sector público.

\begin{abstract}
The low level of strategic alignment of IT that exists in many entities of the public sector causes these organizations do not reach a high performance, in spite of the implementations of practices and of the management given by the government of IT. For this reason, studies have been made of the factors that influence the implementation of IT governance and its areas, among which the strategic alignment of IT stands out. However, the factors that influence each of the practices that lead to strategic alignment have not yet been identified, which is important to know as each practice shapes the IT strategy of the organization, and this will allow taking actions to mitigate the factors negative or enhance the positive ones, and as a consequence obtain a good strategic alignment. In this work we have identified 16 implementation factors, 10 practices for the strategic alignment of IT, in the context of the public sector, and 48 influence relationships (factor $\rightarrow$ practice). An empirical study on 50 executives from the public sector validates 41 of the 48 relationships proposed with a rating between "High" and "Very High", at 95\% confidence through the t-Student test.
\end{abstract}

Keywords: IT alignment strategic; IT governance; practices; factors; public sector.

Díaz, J., Mauricio, D. (2019) Factores que influyen en implementación de prácticas para el alineamiento estratégico de TI en el sector público. Revista Peruana de Computación y Sistemas, 2(2):3-24. http://dx.doi.org/10.15381/rpcs.v2i2.17225

(C) Los autores. Este artículo es publicado por la Revista Peruana de Computación y Sistemas de la Facultad de Ingeniería de Sistemas e Informáticade la Universidad Nacional Mayor de San Marcos. Este es un artículo de acceso abierto, distribuido bajo los términos de la licencia Creative Commons Atribucion - No Comercia_Compartir Igual 4.0 Internacional. (http://creativecommons.org/licenses/by-nc-sa/4.0/) que permite el uso no comercial, distribución y reproducción en cualquier medio, siempre que la obra original sea debidamente citada. 


\section{Introducción}

Las organizaciones del sector público tienen como uno de sus fines el prestar mayores y mejores servicios públicos a los ciudadanos [1], siendo la tecnología de la información (TI) una herramienta crítica para la ejecución y conducción de dichos servicios [2]. En ese sentido, la preocupación de las organizaciones radica en alinear las TI con el negocio y, por consiguiente, sus estrategias, esto es denominado como alineamiento de TI [26], o alineamiento de TI y negocios [42], alineamiento estratégico de TI [7]; en adelante se le denominará ATI.

ATI también es conceptualizado como el ajuste o sincronización entre las estrategias de TI con las estrategias del negocio [3], y desde hace tiempo es la primera preocupación de los ejecutivos de las organizaciones alrededor del mundo [4], pero en el sector público existe una brecha aún por investigar [5].

En la literatura se han encontrado diferentes enfoques y modelos para soportar ATI debido a su complejidad y entendimiento holístico $[69,9,12]$, y es considerado un objetivo primordial del gobierno de TI (GTI) [7].

Para llevar a cabo un GTI efectivo orientado al logro del ATI, la industria y la academia han desarrollado estándares y marcos como la ISO/IEC 38500, COBIT, ITIL, Mecanismos de GTI, entre otros. Cada uno de estos reúne las mejores prácticas de TI y de gestión enfocadas al proceso de ATI; a la cual en el presente se denomina prácticas de alineamiento estratégico de TI (PATI). Sin embargo, en la implementación de estas prácticas, las organizaciones vienen enfrentando dificultades, a pesar de que las prácticas son universalmente conocidas, pero no son aplicadas en las organizaciones [8]. Como resultado de esto, en el caso del sector público a nivel de Latinoamérica, la implementación de prácticas de gobierno de TI (dentro las cuales se encuentra las que conducen al alineamiento estratégico de TI) todavía no ha alcanzado el nivel de otros continentes, ya que se encuentra en una etapa de desafío y creciente [8]. Ante este escenario, surge la pregunta si existen factores que influyen en la implementación de prácticas de alineamiento estratégico de TI.

Los factores han sido estudiados teniendo en cuenta su importancia y su contribución al logro del desempeño de gobierno de TI y de la organización en [25,52]; y desde el punto de vista de la implementación del GTI, considerando factores internos y externos a la organización [6]. En [27], se ha estudiado factores en adopción de marcos de GTI, a partir de las teorías de modelos de adopción de la innovación; y en [10] la adopción de prácticas de GTI, pero desde un punto de vista general y bajo el contexto organizacional. Existen estudios de factores en forma de inhibidores de GTI [29,57], así como factores que influyen dentro de las áreas específicas de gobierno de TI, como el ATI [11]. Sin embargo, ningún estudio previo ha considerado la influencia de los factores sobre cada una de las prácticas de GTI que conducen al ATI (Factores $\rightarrow$ Prácticas) en el sector público, lo cual es necesario conocer para implementar adecuadamente las prácticas que conduzcan al éxito del ATI.

El propósito de este trabajo es identificar qué factores influyen en la implementación de cada práctica de ATI en el sector público, para este fin se estudian las prácticas de ATI y los factores que influyen en ella, se construye un modelo conceptual que establece 48 relaciones entre 16 factores y 10 prácticas identificadas.

Este artículo está organizado como sigue. En la sección 2, se describe la revisión de la literatura de factores y prácticas de alineamiento estratégico de TI. En la sección 3, se propone un modelo conceptual. La validación del modelo sigue una metodología dada en la sección 4. Los resultados y la discusión son desarrollados en la sección 5. Finalmente, las conclusiones siguen en la sección 6.

\section{Revisión Literaria}

\subsection{Prácticas de GTI para el ATI en el sector público}

Alineamiento estratégico de TI es el compromiso conjunto de todas las estrategias, planes de procesos, inversiones y decisiones de las unidades de TI para respaldar la funcionalidad general y el propósito de los objetivos y metas de la organización [13].

A raíz del auge de las TI, el GTI extiende su dominio de la parte tecnológica al negocio [2]. En la actualidad, el GTI viene soportando y conduciendo el ATI, para el cual la academia y la industria han creado estándares y marcos de trabajo, por ejemplo, el estándar ISO/ IEC 38500 a través del consejo directivo conduce el ATI [70]; COBIT 4.1 que en su dominio "PO planear y organizar" contempla el ATI [44,7]; y COBIT 5, que es enfocado en el gobierno corporativo o empresarial, contempla al ATI como un objetivo de todo el marco [14]; y los marcos de trabajo denominados mecanismos de gobierno de TI $[15,16]$. Cada uno de estos estándares y marcos contemplan un conjunto de buenas prácticas de GTI, para el alineamiento de TI.

En [17], se identifica 32 prácticas de GTI, se evalúa su madurez, y se descubre que las prácticas con un score de nivel de madurez igual o mayor a 2 mejora el ATI. En forma similar, en [18], identifica 33 prácticas de GTI en el nivel estratégico y en el nivel operacional. En [37], describe prácticas actuales de GTI para el ATI, y problemas y consecuencias encontrados en el sector público de Tanzania. En [19], se propone un modelo, donde las prácticas claves generan ATI y valor del negocio y halló que las prácticas como el "Comité directivo de TI", "Liderazgo y apoyo de la alta dirección en TI", y la "Gestión de portafolio" contribuyen al ATI y este a su vez impacta en la generación de valor público.

Una relación de 30 prácticas para el ATI se muestra en la tabla A del apéndice. 


\subsection{Factores que influyen en la implementación de prácticas de ATI en el sector público}

Las organizaciones, en el proceso de soportar o conducir ATI, requieren implementar prácticas de alineamiento, en ese proceso han encontrado diversos factores de tipos institucionales, políticos y otros, que no permiten una buena implementación [24,25]. Estos factores son características, condiciones o variables que, cuando se sostienen, mantienen o administran adecuadamente, pueden tener un impacto significativo en el éxito de una empresa que compite en la industria [23].

Existen factores que son de influencia positiva y son denominados de drivers, conductores y facilitadores $[26,27,28]$, y de influencia negativa también nombrado de inhibidores, barreras, obstáculos [29,30,31].

En [29], se identificó cinco factores que inhiben la implementación de GTI: falta de comunicación, inadecuado involucramiento de los interesados, falta de principios y políticas claros de Gobierno de TI, falta de procesos, e inadecuado soporte de recursos financieros. De igual forma, en [33], se muestra que las organizaciones del sector público de Corea del Sur exhiben más barreras en comparación con el privado, identificando varios factores, entre los que destacan la falencia de conocimiento y habilidades, conciencia, tiempo, y recursos financieros. Del mismo modo, en [34], se identifica los factores con alto score: involucramiento del alto nivel, y el soporte para efectividad del gobierno; y con menor score: la medición del desempeño. Un estudio en la India que categoriza los factores en recursos, capacidad y visión cultural de la organización es llevado a cabo en [26]. Y en el sector público de Tanzania, el estudio dado en [11] identifica 11 factores para el ATI, la gestión de riesgos, la entrega de valor y la gestión de recursos, en el ámbito interno de la organización. También, en [24], se propone un marco de trabajo para implementación de GTI, tomando en cuenta factores para ATI, y que es basado en COBIT y la ISO 38500.

Una lista de 52 factores identificados en la presente revisión se encuentra en la tabla B del apéndice.

\section{Modelo Propuesto}

\subsection{Motivación}

Diversos estudios se han preocupado por identificar los factores que afectan al logro de alineamiento de TI en forma general y conjunta o visto desde sus dimensiones o áreas respectivas, pero existe aún la necesidad de conocer cuáles son los factores que afectan a las prácticas específicas, de forma que se puedan establecer estrategias para mitigar los factores inhibidores o potenciar los factores facilitadores, y con ello tener éxito en el ATI. Se propone un modelo conceptual que relaciona 16 factores y 10 prácticas para el ATI, con un total de 48 relaciones (48 hipótesis).

\subsection{Prácticas para el ATI}

A partir de la revisión de la literatura, se han identificado diversas prácticas para el ATI (ver tabla A), por ello se ha establecido los siguientes criterios para seleccionar prácticas aplicables al sector público dadas en el anexo:

- Prácticas que muestran evidencia de uso en el sector público.

- Prácticas mencionadas en dos o más estudios bajo el contexto del sector público.

Considerando los criterios mencionados, se ha seleccionado 10 prácticas para el ATI, que se muestran en la tabla 1. La práctica P01 incluye las prácticas de comités directivos de TI, sean estos de proyectos tácticos o estratégicos.

Las prácticas para el ATI, de acuerdo a [15], se pueden agrupar en tres dimensiones: estructuras, procesos y relaciones. Estructuras, son responsables de definir roles y responsabilidades para la toma de decisiones en la organización, ya sea a través de comités directivos compuestos de ejecutivos de TI y negocios $[78,56]$. Procesos, es el planeamiento y toma de decisiones estratégicas de TI, que incluyen el objetivo de alinear TI con el negocio y el buen desempeño $[78,56]$. Lo relacional abarca la participación activa y colaborativa entre TI y los negocios, empleando la buena comunicación, y el conocimiento y aprendizaje compartido [78,56]. De la tabla 1, las prácticas P01-P03, P04-P08 y P09-P10 corresponden a las dimensiones estructuras, procesos y relacional, respectivamente, de acuerdo al modelo de implementación de GTI [15,16].

\subsection{Factores de Implementación}

De acuerdo a lo revisado, existen factores que influyen en el ATI. Sin embargo, no existen estudios que muestren su influencia en cada una de las prácticas de ATI en el sector público. Para identificar los factores que influyen en las prácticas seleccionadas (ver tabla 1), se consideró el siguiente procedimiento:

- Primero, se ha construido a partir de los factores (ver tabla B) y las prácticas seleccionadas (tabla 1), una matriz de factores $\mathrm{x}$ prácticas (factores $\rightarrow$ prácticas).

- Segundo, se realizó una calificación para cada relación factor $\rightarrow$ práctica, considerando un valor de 1 a 5 para la influencias del factor sobre la práctica, como sigue: $1=$ "Nada", 2 = "Poco", 3 = "Medio", 4 = "Alto", 5 = "Muy alto". Por ejemplo, la relación "Cultura organizacional" $\rightarrow$ "Comité directivo de TI" tiene una calificación de $5=$ "Muy alto", debido a que una cultura organizacional apropiada permitirá la implementación de prácticas necesarias para llevar a cabo el gobierno de TI, siendo una ellas la conformación del "Comité directivo de TI". El detalle de esta calificación se encuentra en [79].

- Tercero, se ha seleccionado las relaciones que tienen calificaciones de "Alto" y "Muy alto". 
La aplicación de este procedimiento permitió identificar 16 factores (ver tabla 2) de los 52 factores que se muestran en el anexo (ver tabla B), y 48 relaciones que se explican en la siguiente sección. Los factores
"Madurez de TI" y "Competencia organizacional de TI" (tabla B) se encuentran individuales, pero por la definición dada por [65], la competencia de TI recoge las capacidades y características relevantes a la madurez de TI,

Tabla 1. Prácticas de ATI seleccionadas

\begin{tabular}{|c|c|c|}
\hline Prácticas & Descripción & Fuente \\
\hline P01: Comité directivo de TI & $\begin{array}{l}\text { Comité en el nivel de la alta dirección, responsable para determinar prioridades de negocios e } \\
\text { inversión en TI. }\end{array}$ & {$[22,5,20]$} \\
\hline P02: $\mathrm{ClO}$ en la mesa directiva & $\begin{array}{l}\text { El ClO participa en la toma de decisiones de alto nivel de la organización, permitiendo que el } \\
\text { liderazgo de TI contribuya al logro organizacional. }\end{array}$ & {$[20,37]$} \\
\hline $\begin{array}{l}\text { P03: Roles y responsabilidades en } \\
\text { gobierno de TI }\end{array}$ & $\begin{array}{l}\text { Establecimiento de roles y responsabilidades, para personal de mando encargado de conducir } \\
\text { el GTI, a fin de participar en la estrategia de la institución y de la estrategia de TI interna, sean } \\
\text { estos personal de TI y Negocios. }\end{array}$ & {$[20,21]$} \\
\hline $\begin{array}{l}\text { P04: Sistema de Medición del } \\
\text { desempeño }\end{array}$ & $\begin{array}{l}\text { Medición del desempeño en dominios de contribución empresarial, orientación de usuario, } \\
\text { operacional, y orientación futura. }\end{array}$ & {$[22,80,81]$} \\
\hline $\begin{array}{l}\text { P05: Metodología de Gestión de } \\
\text { Proyectos de TI }\end{array}$ & $\begin{array}{l}\text { Procesos (estandarizados) internos para dirigir el desarrollo de los proyectos de } \mathrm{TI} \text {, orientados } \\
\text { al logro de los objetivos del negocio. }\end{array}$ & {$[39,21,81]$} \\
\hline $\begin{array}{l}\text { P06: Planeamiento estratégico } \\
\text { de TI }\end{array}$ & $\begin{array}{l}\text { Proceso para definir la estrategia de TI de la organización enfocado en los procesos de negocios } \\
\text { y al logro de objetivos de la organización. }\end{array}$ & {$[20,21,76]$} \\
\hline P07: Gestión de Portafolio de TI & $\begin{array}{l}\text { Proceso de priorización de proyectos (TI) institucionales, que incluye inversiones y en la que el } \\
\text { área de negocios está involucrada. }\end{array}$ & {$[21,19]$} \\
\hline P08: Gestión de Inversión de TI & $\begin{array}{l}\text { Procedimiento estándar para gestionar las inversiones (proyectos de } \mathrm{TI} \text { ). Cómo se realizan los } \\
\text { gastos de } \mathrm{TI} \text {, ejecución, reporte de presupuesto y/o monitoreo, medición del valor (costo/be- } \\
\text { neficio). }\end{array}$ & {$[5,21,81]$} \\
\hline $\begin{array}{l}\text { P09: Rotación de trabajo y entrena- } \\
\text { miento multifuncional (TI/negocio) }\end{array}$ & Personal de TI y de negocios trabajando y capacitándose entre las áreas de TI y de negocios. & {$[20,37]$} \\
\hline $\begin{array}{l}\text { P10: Sistemas de Comunicación } \\
\text { Interna corporativa }\end{array}$ & Comunicación interna entre equipo de negocios y de TI, para tratar asuntos de ambas áreas. & {$[22,37]$} \\
\hline
\end{tabular}

Tabla 2. Factores de influencia en la implementación de prácticas de ATI

\begin{tabular}{|c|c|c|}
\hline Factores & Descripción & Fuente \\
\hline $\begin{array}{l}\text { F01: Adecuado involucramiento } \\
\text { de la alta dirección }\end{array}$ & $\begin{array}{l}\text { Compromiso de la alta dirección con el proceso de adopción del GTI, en términos de su partici- } \\
\text { pación y voluntad de asignar valiosos recursos de la organización. }\end{array}$ & $\begin{array}{l}{[11,22,30} \\
27,62]\end{array}$ \\
\hline F02: Cultura organizacional & $\begin{array}{l}\text { Representa los valores, creencias y principios de la organización que sirven de base para el } \\
\text { sistema de gestión de la organización. }\end{array}$ & {$[24,55,30,46]$} \\
\hline $\begin{array}{l}\text { F03:Estructura de gobierno } \\
\text { corporativo }\end{array}$ & $\begin{array}{l}\text { Estructura orgánica de la organización para la toma de decisiones, que puede ser horizontal, } \\
\text { jerárquica o mixta. }\end{array}$ & [53], [54] \\
\hline $\begin{array}{l}\text { F04: Madurez y competencia } \\
\text { Organizacional de TI }\end{array}$ & $\begin{array}{l}\text { Nivel de competencia y experiencia adecuada para la gestión de TI y para situar a las TI como } \\
\text { clave en la entrega de beneficios a la organización. }\end{array}$ & {$[53,55]$} \\
\hline F05:Política Organizacional & $\begin{array}{l}\text { Conjunto de actividades para la toma decisiones que afectan a toda la organización y que, en } \\
\text { general, son dadas en directivas, cuya finalidad es hacer que la organización alcance sus metas. }\end{array}$ & {$[33,40]$} \\
\hline F06:Disponibilidad al cambio & $\begin{array}{l}\text { Apertura y disposición a adoptar nuevos procesos innovativos, que pueden haber sido resis- } \\
\text { tidos por un fuerte rechazo al cambio, estos cambios implican nueva forma de trabajo en la } \\
\text { organización. }\end{array}$ & {$[77,72]$} \\
\hline $\begin{array}{l}\text { F07: Buena relación entre perso- } \\
\text { nal de TI y negocios }\end{array}$ & $\begin{array}{l}\text { Participación conjunta del personal ejecutivo de TI y negocios en la formulación y ejecución de } \\
\text { las estrategias de negocios y de TI, para alcanzar las metas de la organización. }\end{array}$ & {$[58,56,42]$} \\
\hline $\begin{array}{l}\text { F08: conocimiento de dominio } \\
\text { compartido entre personal de TI } \\
\text { y negocios }\end{array}$ & $\begin{array}{l}\text { Este constructo refleja la forma en que TI entiende el lado del negocio y a la vez la forma en que } \\
\text { el área de negocios entiende el lado de TI, lo que permite a cada una de las áreas a compartir } \\
\text { una visión estratégica de los procesos de cada área; lo cual está orientado al logro de los obje- } \\
\text { tivos organizacionales. }\end{array}$ & {$[16,50$,} \\
\hline F09: Liderazgo de TI & $\begin{array}{l}\text { Capacidad para influenciar y persuadir en las áreas de negocios, para proponer las mejores } \\
\text { alternativas competitivas del mercado a la hora de implementar soluciones de TI. }\end{array}$ & {$[11,42,56]$} \\
\hline $\begin{array}{l}\text { F10: Ambiente regulatorio y } \\
\text { requerimiento de cumplimiento }\end{array}$ & $\begin{array}{l}\text { Leyes, regulaciones y políticas gubernamentales o privadas proveen apoyo a los nuevos negocios, y } \\
\text { reducen el riesgo para las organizaciones, además, en general, son de carácter obligatorio. }\end{array}$ & $\begin{array}{l}{[14,24,28,27,} \\
45]\end{array}$ \\
\hline $\begin{array}{l}\text { F11: Suficiente soporte finan- } \\
\text { ciero }\end{array}$ & $\begin{array}{l}\text { Adecuado apoyo financiero para implementación de proyectos de TI, ya que implican adqui- } \\
\text { sición de infraestructura de TI, contratación de servicios, personal, adquisición de licencias de } \\
\text { software, y otros activos. }\end{array}$ & {$[29,30,24,28]$} \\
\hline $\begin{array}{l}\text { F12:Adecuado equipo y capaci- } \\
\text { dades de TI }\end{array}$ & $\begin{array}{l}\text { Gestión de las capacidades del personal de TI, como las habilidades del equipo, el tamaño y las } \\
\text { calificaciones necesarias; además de atraer, desarrollar y retener profesionales competitivos en } \\
\text { TI. }\end{array}$ & $\begin{array}{l}{[14,24,27} \\
33,45]\end{array}$ \\
\hline F13:Complejidad de TI & $\begin{array}{l}\text { Dificultad intelectual asociada a la comprensión de una innovación (entiéndase la TI como de } \\
\text { innovación tecnológica), pues requiere de la expertise, aceptación y adaptación. }\end{array}$ & {$[31,33,51]$} \\
\hline $\begin{array}{l}\text { F14:Dificultad en demostrar } \\
\text { valor y beneficios }\end{array}$ & $\begin{array}{l}\text { Falta de evidencia de mostrar los beneficios anticipados y posteriores de implementar proyec- } \\
\text { tos de TI (metodologías, nuevas prácticas, entre otros). }\end{array}$ & {$[30,31,51]$} \\
\hline $\begin{array}{l}\text { F15:Soporte externo de Provee- } \\
\text { dores y consultorías }\end{array}$ & $\begin{array}{l}\text { Adecuado apoyo técnico de entidades externos (proveedores), en la implementación de nuevos } \\
\text { proyectos de TI. }\end{array}$ & {$[27,31,83]$} \\
\hline $\begin{array}{l}\text { F16:Clara estrategia de TI, prin- } \\
\text { cipios y políticas }\end{array}$ & $\begin{array}{l}\text { Establecimiento de las directivas internas para que las } \mathrm{TI} \text { apoyen al logro de objetivos organiza- } \\
\text { cionales. La disposición rige para todas las unidades (negocios y } \mathrm{TI} \text { ) de la organización. }\end{array}$ & $\begin{array}{l}{[11,24,29,} \\
45]\end{array}$ \\
\hline
\end{tabular}


por lo que se presenta como un solo factor "Madurez y competencia organizacional de TI” F04.

\subsection{Hipótesis}

Además de las relaciones factor $\rightarrow$ práctica con calificaciones de "Alta" y "Muy Alta" establecidas en la sección anterior, se procedió a sustentar dichas relaciones.

3.4.1 Adecuado Involucramiento de TI por parte de la alta dirección (F01): En un reporte del Instituto de gobierno de TI ITGI [8], sobre el estado de gobernanza empresarial de TI en las empresas, encontró que la "falta de compromiso y apoyo de la alta dirección" es un desafío en la implementación del gobierno de TI [61]. Según [17], las prácticas P01, P02, P03 son las que están presentes en las estructuras de gobierno de TI. Además, al ser estas prácticas claves para el inicio del gobierno, deben ser implementadas con el "Adecuado involucramiento por parte de la alta dirección"; y no solo conducidas por las áreas técnicas o apoyadas por un nivel jerárquico medio o inferior, de lo contrario fracasarían en su intento de implementarlas. Por lo que se plantea las siguientes hipótesis:

- H1.1: El adecuado involucramiento de TI por parte de la alta dirección influye positivamente a la implementación del "Comité directivo de TI".

- H1.2: El adecuado involucramiento de TI por parte de la alta dirección influye positivamente a la implementación del "CIO en la mesa Directiva".

- H1.3: El adecuado involucramiento de TI por parte de la alta dirección influye positivamente a la asignación de "Roles y responsabilidades para el gobierno de TI".

3.4.2 Adecuado cultura organizacional (FO2): Según [32], la cultura organizacional tiene influencia significativa en las estructuras de gobierno de TI. Las prácticas P01, P02 y $\mathrm{P} 03$ son las que pertenecen a las estructuras del gobierno de TI [18], y su implementación se da con el apoyo del alto nivel jerárquico quien promueve la adopción de nuevas prácticas en la organización. Por lo que se propone las hipótesis:

- H2.1: Una adecuada "Cultura organizacional" influye positivamente a la implementación del "Comité directivo de TI".

- H2.2: Una adecuada "Cultura organizacional" influye positivamente a la implementación del "CIO en la mesa Directiva".

- H2.3: Una adecuada "Cultura organizacional" influye positivamente a la asignación de "Roles y responsabilidades para asignar tareas de integración en alineamiento y gobierno".

La cultura organizacional además afecta a todas las actividades colaborativas de la organización, en particular a las actividades que se realizan en forma conjunta entre el área de TI y el negocio, es decir, afecta a las prácticas P09 y P10. Por lo que se propone las hipótesis:
- H2.9: La adecuada "Cultura organizacional" influye en la implementación de la práctica "Rotación de trabajo y entrenamiento multifuncional (TI/negocio)".

- H2.10: La adecuada "Cultura organizacional" influye en la implementación de un "Sistema de comunicación interna corporativa".

\subsubsection{Estructura de gobierno corporativo} (F03): El uso de las tecnologías de la información ha cambiado la forma de realizar las actividades y los procesos en las organizaciones y, por ende, la forma de sus estructuras organizacionales sufren cambios en la actualidad [55]. Estos cambios implican considerar personal de TI en la toma de decisión a través de un comité directivo de TI, en la cual se requiere que el CIO (gerente de TI) participe. Por lo que se plantea las siguientes hipótesis:

- H3.1: La "Estructura de gobierno corporativo" influye en la conformación del "Comité directivo de TI".

- H3.2: La "Estructura de gobierno corporativo" influye a la implementación del "CIO en la mesa directiva".

3.4.4 Madurez y competencia organizacional de TI (F04): La madurez de TI implica que la organización tiene dominio y experiencia en las tomas de decisiones de TI y alienados al negocio. Por otro lado, la competencia organizacional de TI implica que exista una estructura organizacional de TI que permita que las decisiones sobre TI se concreten. Por consiguiente, la organización podrá implementar un comité directivo de TI (P01), y tener en dicho comité un CIO (P02), además de establecer los "Roles y responsabilidades en el gobierno de TI" (P03), siendo una de las responsabilidades el "Planeamiento estratégico de TI" (P06). Por ello se formula las siguientes hipótesis:

- H4.1: La suficiente "Madurez y competencia organizacional de TI" afecta positivamente a la implementación de un "Comité directivo de TI".

- H4.2: La suficiente "Madurez y competencia organizacional de TI" afecta positivamente a la implementación del "CIO en la mesa directiva"..

- H4.3: La suficiente "Madurez y competencia organizacional de TI" afecta positivamente a la implementación de los "Roles y responsabilidades para el gobierno de TI".

- H4.6: La suficiente "Madurez y competencia organizacional de TI" afecta positivamente a la implementación del "Planeamiento estratégico de TI".

3.4.5 La Politica Organizacional (F05): De acuerdo a un reporte de PWC [63], la política organizacional debe ejercerse para la adopción e implementación de prácticas de gobierno de TI.

Las prácticas P01, P03 se verán influenciados por F05 debido a que estas prácticas son las que forman parte de la estructura del gobierno de TI y para implemen- 
tarse requiere que la organización haya adoptado como política la conducción del gobierno de TI. Por lo que se plantea las siguientes hipótesis:

- H5.1: La adecuada "Política organizacional" afecta positivamente a la implementación del "Comité directivo de TI".

- H5.3: La adecuada "Política organizacional" afecta a la asignación de "Roles y responsabilidades en el gobierno de TI".

La política de la organización toma en cuenta las relaciones entre las áreas de TI y negocios, es decir, influye en las prácticas que tienen relaciones directas entre ambas áreas. Por lo que se propone las hipótesis:

- H5.9: La adecuada "Política organizacional" afecta positivamente para implementar la "Rotación de trabajo y entrenamiento multifuncional (TI/negocio)".

- H5.10: La adecuada "Política organizacional" afecta positivamente para implementar un "Sistema de comunicación interna corporativa".

3.4.6 Disponibilidad al cambio (F06): La implementación de nuevos procesos que modifican la (forma cotidiana de realizar actividades) forma de trabajo requiere de la disposición y la aceptación al cambio por parte de todo el personal ejecutivo y especialistas de la organización, incluso cuando estas formas de trabajo afectan al movimiento del personal hacia otras áreas, donde requiere de adaptación y aprendizaje continuo. Esta disposición permite que las unidades técnicas conozcan las necesidades, desafíos y nuevas ideas de negocio que la organización pueda plantear. Por lo que se propone la hipótesis:

- H6.9: La "Disponibilidad al cambio" afecta positivamente a la implementación de "Rotación de trabajo y entrenamiento multifuncional (TI/negocio)".

3.4.7 Buena relación entre personal de TI y negocios (F07): La implementación exitosa de las TI influye a la estrecha comunicación entre personal de TI y negocios [64]. Esto sucede también de forma inversa, de modo que una estrecha relación entre el personal de TI y negocios influye en la implementación de TI, principalmente en lo relacionado a la TI y la comunicación. Por lo que se propone la hipótesis:

- H7.10: La "Buena relación entre personal de TI y negocios" influye a la implementación de un "Sistema de comunicación interna corporativa".

P09 se caracteriza por tener la participación activa del personal de TI y negocios, con el fin de adquirir el conocimiento de las actividades y procesos de ambas áreas; y la buena relación entre ambos permitirá el éxito de la implementación de la "Rotación de trabajo y el entrenamiento cruzado". Por lo que se propone la hipótesis:
- H7.9: La "Buena relación entre los ejecutivos de TI y negocios" influye en la implementación de la "Rotación de trabajo y entrenamiento multifuncional (TI/ negocio)".

\subsubsection{Conocimiento de dominio compartido} entre TI y negocios (F08): El conocimiento que posee un área sobre la otra despierta el interés del personal, $y$, cuando el conocimiento es compartido, permite la participación mutua entre las unidades de TI y de negocios en los procesos claves de cada uno [60], por lo que este afecta positivamente a la "Rotación de trabajo y entrenamiento cruzado (P09)”. Además, influenciará positivamente a la comunicación entre ejecutivos de TI $y$ de negocios [64], y, por consiguiente, en la implementación de un "Sistema de comunicación interna" (P10), con el propósito de lograr el buen desempeño entre las áreas involucradas. Por lo que se propone las hipótesis:

- H8.9: El "Conocimiento de dominio compartido entre TI y negocios" influye positivamente a la implementación de la "Rotación de trabajo y entrenamiento multifuncional (TI/negocio)".

- H8.10: El "Conocimiento de dominio compartido entre TI y negocios" influye positivamente a la implementación del "Sistema de comunicación interna corporativa".

\subsubsection{Liderazgo de TI (F09): El Liderazgo} que ejerce TI frecuentemente se da cuando se implementa productos innovadores que agilizan los procesos dentro de la organización [42]. Este liderazgo se encarga de influenciar, persuadir y comunicar a las demás áreas de la organización acerca de los nuevos proyectos de TI, y, para que sea más efectivo, este influenciará en implementar el "Sistema de comunicación interna corporativa", permitiendo comunicar todas las iniciativas, propuestas, estado de los avances, metas, logros de los proyectos, y la retroalimentación de todas las áreas de la organización. Por lo que se propone la hipótesis:

- H9.10: El "Liderazgo de TI" afecta positivamente a la implementación de un "Sistema de comunicación interna corporativa".

3.4.10 Ambiente Regulatorio y requerimiento de cumplimiento (F10): Las normas regulatorias de TI en el sector público se emiten a través de instituciones gubernamentales, agencias internacionales, empresas multinacionales, instituciones financieras y organizaciones de trabajo [67]. Estas son obligatorias y, en general, consideran la implementación del "Planeamiento estratégico de TI", así como de la "Metodología de gestión de proyectos de TI". Por lo que se plantea las siguientes hipótesis:

- H10.5: El adecuado "Ambiente regulatorio y requerimiento de cumplimiento" influye positivamente a la implementación de una "Metodología de gestión de proyectos de TI". 
- H10.6: El adecuado "Ambiente regulatorio y requerimiento de cumplimiento" afecta positivamente a la implementación del "Planeamiento estratégico de TI".

3.4.11 Suficiente soporte financiero (F11): El presupuesto inadecuado para recursos requeridos de TI es uno de los principales problemas encontrados en las prácticas de gobierno de TI en las organizaciones [37], por lo que es necesario para el éxito de los proyectos de TI y, por consiguiente, este afecta a la implementación de las prácticas "Planeamiento estratégico de TI", "Sistema de Medición del desempeño" y a la "Metodología de gestión de proyectos de TI". Por lo que se propone las hipótesis:

- H11.4: El "Suficiente soporte financiero" afecta positivamente a la implementación del "Sistema de medición del desempeño".

- H11.5: El "Suficiente soporte financiero" influye positivamente en la implementación de una "Metodología de gestión de proyectos de TI".

- H11.6: El "Suficiente soporte financiero" afecta positivamente a la implementación de la "Planeamiento estratégico de TI".

3.4.12 Adecuado equipo y capacidades de TI (F12): Un reporte de ITGI muestra que la falta de capacidades del personal de TI es una de las dificultades de ATI [8]. Inversamente los equipos y personal calificados de TI conocen de "Metodología de gestión de proyectos de TI", el "Planeamiento estratégico de TI", la "Gestión de portafolios", y la "Gestión de inversión de TI", saben de su importancia en el ATI, facilita las labores y mejora la productividad, por ello facilitará su implementación. Por lo que se plantea las siguientes hipótesis:

- H12.5: El "Adecuado equipo y capacidades de TI" influye positivamente a la implementación de una "Metodología de gestión de proyectos de TI".

- H12.6: El "Adecuado equipo y capacidades de TI" influye positivamente a la implementación del "Planeamiento estratégico de TI".

- H12.7: El "Adecuado equipo y capacidades de TI" influye positivamente a la implementación del "Gestión de portafolios de proyectos de TI".

- H12.8: El "Adecuado equipo y capacidades de TI" influye positivamente a la implementación de la "Gestión de inversión de TI".

3.4.13 Complejidad de TI (F13): El gobierno de TI es de naturaleza complejas y dinámica, por estar compuesto de subsistemas interdependientes (procesos, estructuras y relaciones) [56]. También, las prácticas de gobierno de TI son complejas, desde el enfoque de la adopción de la innovación [30], pues para ser empleadas en los procesos de la organización deben pasar por un proceso de adopción.
Siendo las prácticas P04, P05, P06, P07 y P08 de la categoría de los procesos de gobierno de TI $[15,17]$, entonces por lo antes dicho estas son influenciadas por la complejidad de TI.

Por lo que se plantea las siguientes hipótesis::

- H13.4: La "Complejidad de TI" influye a la implementación del "Sistema de medición del desempeño".

- H13.5: La "Complejidad de TI" influye en la implementación de "Metodología de gestión de proyectos TI".

- H13.6: La "Complejidad de TI" influye en la implementación del "Planeamiento estratégico de TI".

- H13.7: La "Complejidad de TI" influye en la implementación de la "Gestión de portafolio de proyectos de TI".

- H13.8: La "Complejidad de TI" influye a la implementación de la "Gestión de inversión de TI".

3.4.14 Dificultad en demostrar el valor y beneficios de TI (F14): Uno de los problemas desde el punto de vista de los ejecutivos de TI es que existe un alto costo y baja rentabilidad con respecto a la inversión realizada en TI [8]. La deficiencia en mostrar resultados de los proyectos de TI permite que las áreas de toma de decisiones de la organización tengan desconfianza para implementar proyectos de TI, sobre todo si son proyectos para la gestión de TI, donde no se evidencian resultados inmediatos, como los que sí se pueden mostrar en la implementación de software de clientes. Por ello, la dificultad de demostrar el valor y beneficio de TI influyen negativamente en la implementación de prácticas relacionadas a la gestión de TI (P04, P05, P07 y P08), aún más porque sus resultados no se evidencian a corto plazo, a pesar que estas traen ahorros de recursos y proyectos alineados a los objetivos organizacionales o sea ATI. Por lo que se plantea las siguientes hipótesis:

- H14.4: La "Dificultad en demostrar el valor y beneficios" influye en la implementación de un "Sistema de medición del desempeño".

- H14.5: La "Dificultad en demostrar el valor y beneficios" influye en la implementación de "Metodología de gestión de proyectos TI".

- H14.7: La "Dificultad en demostrar el valor y beneficios" influyen en la implementación de la "Gestión de portafolio de proyectos de TI".

- H14.8: La "Dificultad en demostrar el valor y beneficios" influyen en la implementación de la "Gestión de inversión de TI".

3.4.15 Soporte externo de proveedores y consultorías (F15): La falta de apoyo y compromiso de los proveedores ha generado dificultades en la implementación de prácticas de gobierno de TI $[30,31]$. El "Planeamiento estratégico de TI" requiere de recursos financiero y 
personal con expertise, habilidades y dominio de conocimientos para planificar. Estos requerimientos los posee mayormente proveedores o consultores externos que, en general, poco poseen las organizaciones del sector público, además, presenta un alto costo mantener especialistas para realizar una labor muy importante pero esporádica. Por lo que se plantea la siguiente hipótesis:

- H15.6 El "soporte externo de proveedores y consultorías" influye positivamente a la implementación del "Planeamiento estratégico de TI".

\subsubsection{Clara estrategia de TI, principios y po-} liticas (F16): Los principios y políticas del gobierno TI derivadas de la dirección estratégica de las organizaciones es un factor muy importante para un GTI exitoso [41], y al mismo tiempo este tiene por rol conducir el ATI. La definición de estrategias, los principios y políticas permite que las prácticas directamente relacionadas con el GTI sean implementadas para cumplir el logro institucional, y siendo P04, P05, P06, P07 y P08 prácticas específicas de GTI, se plantea las siguientes hipótesis:

- H16.4: La "Clara estrategia de TI, principios y políticas" influyen positivamente a la implementación del "Sistema de medición del desempeño".

- H16.5. La "Clara estrategia de TI, principios y políticas" afectan positivamente a la implementación de la "Metodología de gestión de proyectos de TI".

- H16.6. La "Clara estrategia de TI, principios y políticas" influyen positivamente a la implementación del "Planeamiento estratégico de TI".

- H16.7: La "Clara definición de la estrategia de TI, principios y políticas" influyen positivamente a la im- plementación de la "Gestión del portafolio de proyectos de TI".

- H16.8: La "Clara definición de la estrategia de TI, principios y políticas" influyen positivamente a la implementación de la "Gestión de inversión de TI".

La tabla 3 muestra un resumen de las 48 hipótesis propuestas, que son denotadas por Hx.y, donde el factor Fx influye en la implementación de la práctica Py. Así mismo, el modelo conceptual sigue en la figura 1.

\section{Metodología}

\subsection{Recopilación de datos}

En la presente investigación, se desarrolló una encuesta en línea con Survey Google Forms (2018), basada en el modelo propuesto. El objetivo de la encuesta fue determinar la percepción de los entrevistados sobre los factores que influyen en la implementación de prácticas de ATI. La encuesta se estructuró en 2 secciones: i) datos personales y demográficos (4 preguntas), ii) percepción de la influencia de los factores para implementar prácticas de ATI (10 preguntas). Las respuestas a las preguntas de la sección 2 se evaluaron con la escala de Likert de acuerdo con una calificación de cinco valores (1: Nada, 2: Poco, 3: Medio, 4: Alto, 5: Muy Alto). La encuesta se aplicó a los jefes o responsables de la gerencia (oficinas) de informática del sector público, en el periodo de octubre a diciembre de 2018 .

Después de preparar la encuesta, se llevó a cabo una prueba piloto para validar las preguntas, con 8 especialistas en GTI del sector público, quienes verificaron la pertinencia de las preguntas, su formulación y alternativas de respuestas en relación al modelo, luego se hizo las correcciones correspondientes (ver figura 1).

Tabla 3. Matriz de Hipótesis (Factores $\rightarrow$ Prácticas)

\begin{tabular}{|c|c|c|c|c|c|c|c|c|c|c|}
\hline \multirow{2}{*}{ Factores } & \multicolumn{10}{|c|}{ Prácticas } \\
\hline & P01 & P02 & P33 & P04 & P05 & P06 & P07 & P08 & P09 & P10 \\
\hline F01 & $\mathrm{H} 1.1$ & $\mathrm{H} 1.2$ & $\mathrm{H} 1.3$ & & & & & & & \\
\hline F02 & $\mathrm{H} 2.1$ & $\mathrm{H} 2.2$ & $\mathrm{H} 2.3$ & & & & & & $\mathrm{H} 2.9$ & $\mathrm{H} 2.10$ \\
\hline F03 & H3.1 & $\mathrm{H} 3.2$ & & & & & & & & \\
\hline F04 & H4.1 & $\mathrm{H} 4.2$ & $\mathrm{H} 4.3$ & & & $\mathrm{H} 4.6$ & & & & \\
\hline F05 & H5.1 & & $\mathrm{H} 5.3$ & & & & & & H5.9 & $\mathrm{H} 5.10$ \\
\hline F06 & & & & & & & & & H6.9 & \\
\hline F07 & & & & & & & & & $\mathrm{H} 7.9$ & $\mathrm{H} 7.10$ \\
\hline F08 & & & & & & & & & $\mathrm{H} 8.9$ & $\mathrm{H} 8.10$ \\
\hline F09 & & & & & & & & & & H9.10 \\
\hline F10 & & & & & $\mathrm{H} 10.5$ & H10.6 & & & & \\
\hline F11 & & & & $\mathrm{H} 11.4$ & $\mathrm{H} 11.5$ & H11.6 & & & & \\
\hline F12 & & & & & $\mathrm{H} 12.5$ & $\mathrm{H} 12.6$ & $\mathrm{H} 12.7$ & $\mathrm{H} 12.8$ & & \\
\hline F13 & & & & $\mathrm{H} 13.4$ & $\mathrm{H} 13.5$ & H13.6 & $\mathrm{H} 13.7$ & H13.8 & & \\
\hline F14 & & & & $\mathrm{H} 14.4$ & $\mathrm{H} 14.5$ & & $\mathrm{H} 14.7$ & $\mathrm{H} 14.8$ & & \\
\hline F15 & & & & & & H15.6 & & & & \\
\hline F16 & & & & H16.4 & $\mathrm{H} 16.5$ & H16.6 & $\mathrm{H} 16.7$ & $\mathrm{H} 16.8$ & & \\
\hline
\end{tabular}




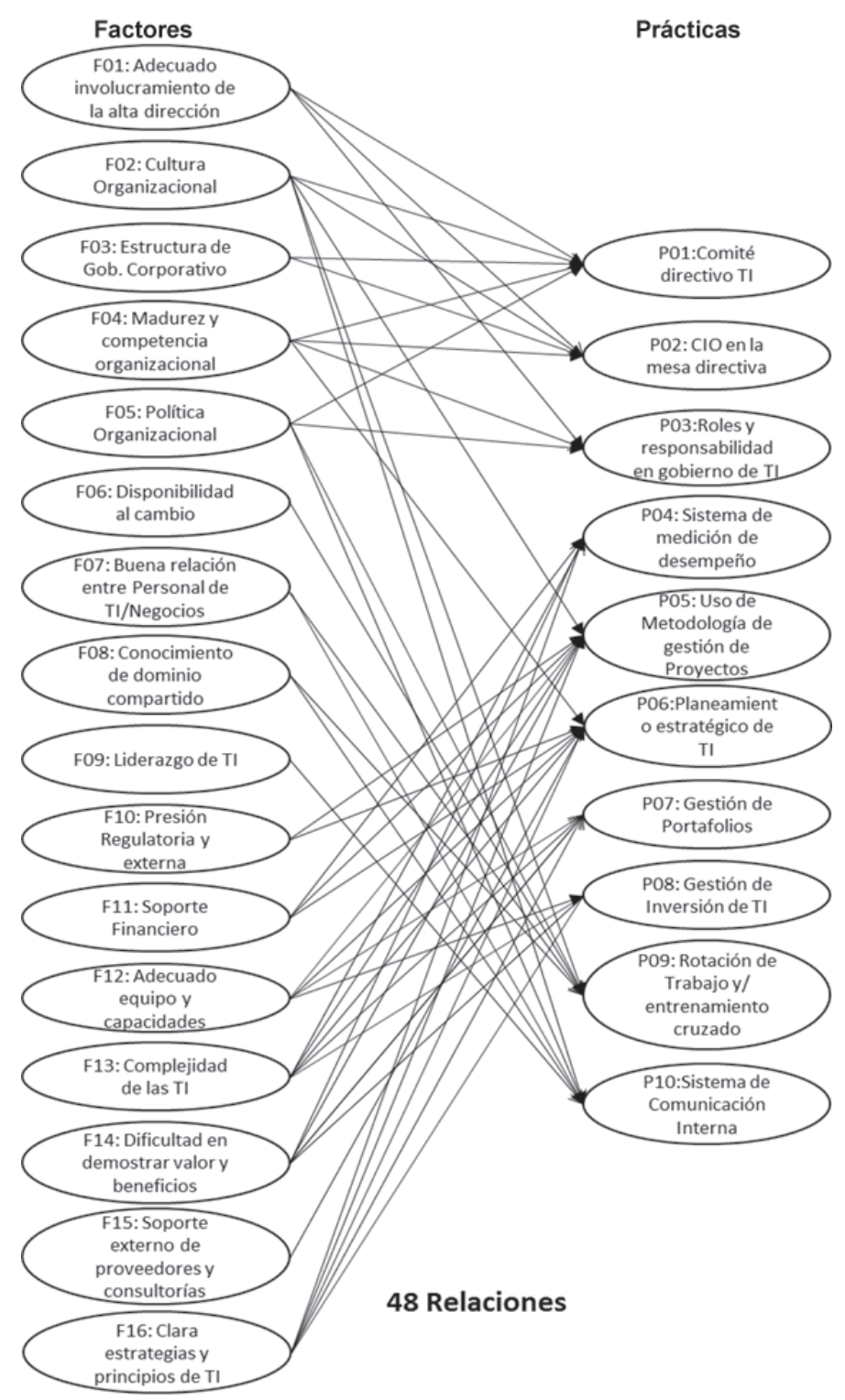

Figura 1. Modelo conceptual (Factores $\rightarrow$ Prácticas)

La población total fue 149 gerencias (oficinas) de informática que conforman el sistema nacional de informática, pertenecientes al poder ejecutivo (exceptuando a las universidades), que son 139, y a los organismos constitucionales autónomos, ubicadas en el departamento de Lima, que son 10 gerencias. Estas entidades se encuentran descritas en el organigrama del Estado peruano del año 2014, que a la fecha permanece vigente y está publicado en el portal web https://www.peru.gob. pe/docs/estado.pdf, a quienes se les remitió cartas para hacerles partícipe de las encuestas. Se obtuvo 50 encuestas respondidas y válidas.

\subsection{Análisis e interpretación de los resultados}

Se ha considerado para el análisis estadístico lo siguiente: i) Prueba de confiabilidad usando el Alfa de Cronbach, ii) Análisis descriptivo de la población (Análisis de aspectos demográficos de la encuesta: tipo de organización, nivel de experiencia y cargo del encuestado, media, varianza, moda y distribución de las res- puestas), iii) Análisis de correspondencia simple (con el fin de conocer el grado de asociación de cada factor de alineamiento con cada práctica), iv) Análisis de correspondencia múltiple (análisis que permite saber el grado en que un factor afecta a más de una práctica), y v) Validación de los resultados a través de prueba de hipótesis t-Student.

\section{Resultados y Discusión}

\subsection{Confiabilidad de datos}

Para estimar la fiabilidad del instrumento de medición, se ha utilizado el Alfa de Cronbach. Cuanto más cercano sea el valor de alfa a 1 , mayor será la consistencia interna de los elementos analizados, y la validez de un instrumento es aceptable si tiene un valor superior a 0.70 [68]. De acuerdo con los resultados obtenidos con la herramienta R, se obtuvo un valor de Alfa de Cronbach igual a 0.94 (ver figura 2). 
Reliability analysis

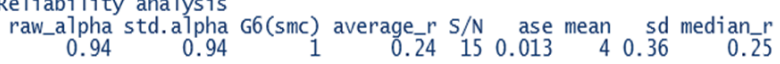

Figura 2. Resultados de "R Studio" sobre la fiabilidad de datos (Factores $\rightarrow$ Prácticas)

\subsection{Análisis descriptivo de la población}

En la tabla 4, se muestra las características de los encuestados, se puede observar que el $88 \%$ de ellos son del poder ejecutivo y $12 \%$ de instituciones autónomas; el $82 \%$ de los encuestados son directores o gerentes de TI, y el $56 \%$ de los encuestados tienen más de 5 a más años de experiencia en el cargo. También de los 7 tipos de entidades del poder ejecutivo han respondido en mayor proporción "los Organismos Públicos Especializados", seguido de los Ministerios.

Se muestra la clasificación de los encuestados en la tabla 4, según el tipo de organización, entidades del poder ejecutivo se obtuvieron un $88 \%$ del total y, por el otro tipo de entidades, los organismos constitucionales autónomos un $12 \%$ del total. Por el tipo de cargo de los encuestados, se obtuvo en mayor proporción a los jefes o gerentes de TI con un $58 \%$. Y, por su experiencia, un $56 \%$ de los encuestados tienen de 6 a más años de experiencia en el cargo.

Tabla 4. Clasificación de encuestados (Factores $\rightarrow$ Prácticas)

\begin{tabular}{|lcc|}
\hline Tipo de Entidades Públicas & $\begin{array}{c}\text { Cant. } \\
(\mathbf{N}=50)\end{array}$ & Porcentaje \\
\hline Org. Constitucionales Autónomos & 6 & $12 \%$ \\
\hline Poder Ejecutivo & 44 & $88 \%$ \\
\hline Consejo de Ministros & 1 & $2 \%$ \\
\hline Empresas & 1 & $2 \%$ \\
\hline Entidades Administrativas de Fondos & 1 & $2 \%$ \\
\hline Intangibles & 15 & $28,6 \%$ \\
\hline Ministerios & 7 & $14 \%$ \\
\hline Org. Públicos Ejecutores & 15 & $31 \%$ \\
\hline Org. Públicos Especializados & 4 & $8 \%$ \\
\hline Programas & 50 & $100 \%$ \\
\hline Rol/Cargo del Responsable & 13 & $26 \%$ \\
\hline Director de TI & 28 & $56 \%$ \\
\hline Jefe o Gerente de TI & 9 & $18 \%$ \\
\hline Sub Gerente o Responsable de TI & 50 & $100 \%$ \\
\hline Experiencia en Años en el Cargo & 22 & $44 \%$ \\
\hline De 0 a 5 & 11 & $22 \%$ \\
\hline De 6 a 10 & 8 & $16 \%$ \\
\hline De 11 a 15 & 9 & $18 \%$ \\
\hline De 16 a más & & \\
\hline
\end{tabular}

Se calculó la media, desviación estándar y moda de la percepción de los encuestados sobre la influencia de los factores en las prácticas, como se observa (tabla C del apéndice), se obtuvo el valor de la "media" de 4 "Alta influencia" en su mayoría, el mínimo valor es de 3.28 y el máximo valor es de 4.8. Con respecto a la desviación estándar, en todos los casos es pequeña, el mínimo es de 0.45 y el máximo de 0.99 . Finalmente, la moda en general es de una puntuación mayor a 4 "influencia alta", excepto por las relaciones H10.5 y H15.6, con moda igual a 3 "influencia media".

\subsection{Análisis de correspondencia simple (ACS)}

Este tipo de análisis permite saber el nivel en que se relaciona cada factor propuesto con cada práctica de alineamiento estratégico de TI (Factor $\rightarrow$ Práctica). En la tabla 5, se muestra los valores propios (eigenvalues) para el cálculo del ACS entre los factores y las prácticas, donde se tiene el valor asociado a la varianza (value), el porcentaje de varianza para cada componente (percentage), y se observa que los dos primeros componentes explican el $70.8 \%$ de los datos de la muestra (43.1\% $+27.7 \%$ ), por ello, se usó los componentes 1 y 2 (Dim 1 y Dim2) y con estos valores se construyó las tablas de inercias que se muestran en las tablas 6 y 7 .

Tabla 5. Eigenvalues para el ACS entre (Factores $\rightarrow$ Prácticas)

\begin{tabular}{lccccccccc}
\hline Component & $\mathbf{1}$ & $\mathbf{2}$ & $\mathbf{3}$ & $\mathbf{4}$ & $\mathbf{5}$ & $\mathbf{6}$ & $\mathbf{7}$ & $\mathbf{8}$ & $\mathbf{9}$ \\
\hline Value & 0.94 & 0.60 & 0.22 & 0.20 & 0.08 & 0.08 & 0.02 & 0.01 & $3 \mathrm{e}-05$ \\
Porcentaje & 43.1 & 27.7 & 10.2 & 9.5 & 4.3 & 3.9 & 3.8 & 0.7 & 0.0 \\
\hline
\end{tabular}

En la tabla 6 se encuentra el grado de contribución de cada factor de ATI a cada uno de los componentes (Dim1, Dim2) y que componente se relaciona más cada factor, la frecuencia total de cada punto (Mass), el valor de la distribución chi cuadrado (ChiDist) y el valor de inercia (inertia). De igual manera, en la tabla 7 se muestra el grado de relación de cada práctica de ATI a cada componente y qué componente está más relacionado.

Además, tomando los datos de las tablas de inercia (tablas 6 y 7), se diseñó la gráfica con plot (figura 3), donde se está considerando la relación (Factor $\rightarrow$ Práctica) con el valor 1 (si se relaciona), cuando la calificación de los encuestados es "igual o mayor 4" y, en caso contrario, el valor 0 (no se relaciona). En este gráfico, se observa, de manera visual, qué "factores" (círculos azules) se relacionan más con las "prácticas" (triángulos rojos), es decir, cuanto más cercanos estén dos puntos significa que están más relacionados.

En la figura 3, se observa las siguientes relaciones simples de cada factor: i) el factor F01 se relaciona con las prácticas P01, P02 y P03, ii) el factor F02 se relaciona con las prácticas P01, P02 y P03, iii) el factor F03 se relaciona con las prácticas P01 y P02, iv) el factor F04 se relaciona con las prácticas P01, P02 y P03, v) el factor F05 se relaciona con las prácticas P09 y P10, vi) el factor F06 se relaciona con la práctica P09, vii) el factor F07 se relaciona con las prácticas P09 y P10, viii) el factor F08 se relaciona con las prácticas P09 y P10, ix) el factor F09 se relaciona con la práctica $\mathrm{P} 10$, x) el factor F10 se relaciona con las prácticas $\mathrm{P} 05$ y $\mathrm{P} 06$, xi) el factor F11 se relaciona con las prácticas P04, $\mathrm{P} 05, \mathrm{P} 06$, xii) el factor F12 se relaciona con las prácticas P05, P06, P07 
Tabla 6. Tabla de inercia de Factores

\begin{tabular}{ccccccccc}
\hline Factor & F01 & F02 & F03 & F04 & F05 & F06 & F07 & F08 \\
\hline Mass & 0.076393 & 0.111406 & 0.044562 & 0.092308 & 0.084881 & 0.023873 & 0.044562 & 0.042971 \\
ChiDist & 1.565 .211 & 0.976580 & 2.015 .556 & 1.166 .676 & 1.162 .587 & 2.801 .743 & 1.842 .323 & 1.842 .299 \\
Inertia & 0.187153 & 0.106249 & 0.181033 & 0.125643 & 0.114725 & 0.187395 & 0.151251 & 0.145846 \\
Dim.1 & -0.834997 & -0.981502 & -0.829422 & -0.379885 & -1.011 .637 & -1.167 .743 & -1.166 .642 & -1.166 .629 \\
Dim.2 & 1.661 .594 & 0.103549 & 1.872 .295 & 1.275 .932 & -0.240427 & -1.866 .594 & -1.845 .132 & -1.844 .867 \\
\hline Factor & F09 & F10 & F11 & F12 & F13 & F14 & F15 & F16 \\
\hline Mass & 0.023342 & 0.031300 & 0.045623 & 0.096552 & 0.081698 & 0.064721 & 0.011141 & 0.124668 \\
ChiDist & 2.779 .722 & 1.774 .388 & 1.516 .108 & 1.245 .761 & 1.127 .100 & 1.428 .196 & 2.472 .490 & 1.079 .000 \\
Inertia & 0.180362 & 0.098546 & 0.104869 & 0.149841 & 0.103785 & 0.132015 & 0.068105 & 0.145144 \\
Dim.1 & -1.165 .541 & 0.935082 & 1.009 .427 & 1.072 .190 & 1.099 .617 & 1.160 .227 & 0.815272 & 1.085 .902 \\
Dim.2 & -1.823 .669 & 0.094096 & -0.039957 & -0.157567 & -0.206728 & -0.314276 & 0.297080 & -0.181563 \\
\hline
\end{tabular}

Tabla 7. Tabla de inercia de Prácticas

\begin{tabular}{ccccccccccc}
\hline Prácticas & P01 & P02 & P03 & P04 & P05 & P06 & P07 & P08 & P09 & P10 \\
\hline Mass & 0.108753 & 0.091247 & 0.091247 & 0.070557 & 0.102918 & 0.140584 & 0.083289 & 0.083820 & 0.112997 & 0.114589 \\
ChiDist & 1.329 .241 & 1.588 .403 & 1.351 .779 & 1.503 .425 & 1.158 .850 & 1.292 .732 & 1.327 .729 & 1.325 .884 & 1.843 .921 & 1.825 .472 \\
Inertia & 0.192154 & 0.230218 & 0.166736 & 0.159479 & 0.138212 & 0.234937 & 0.146827 & 0.147352 & 0.384196 & 0.381850 \\
Dim.1 & -0.826569 & -0.782956 & -0.819270 & 1.125 .644 & 1.102 .078 & 0.790523 & 1.134 .930 & 1.133 .775 & -1.132 .294 & -1.130 .159 \\
Dim.2 & 1.284 .181 & 1.620 .102 & 0.965902 & -0.245174 & -0.192321 & 0.231048 & -0.267277 & -0.264717 & -1.451 .707 & -1.418 .323 \\
\hline
\end{tabular}

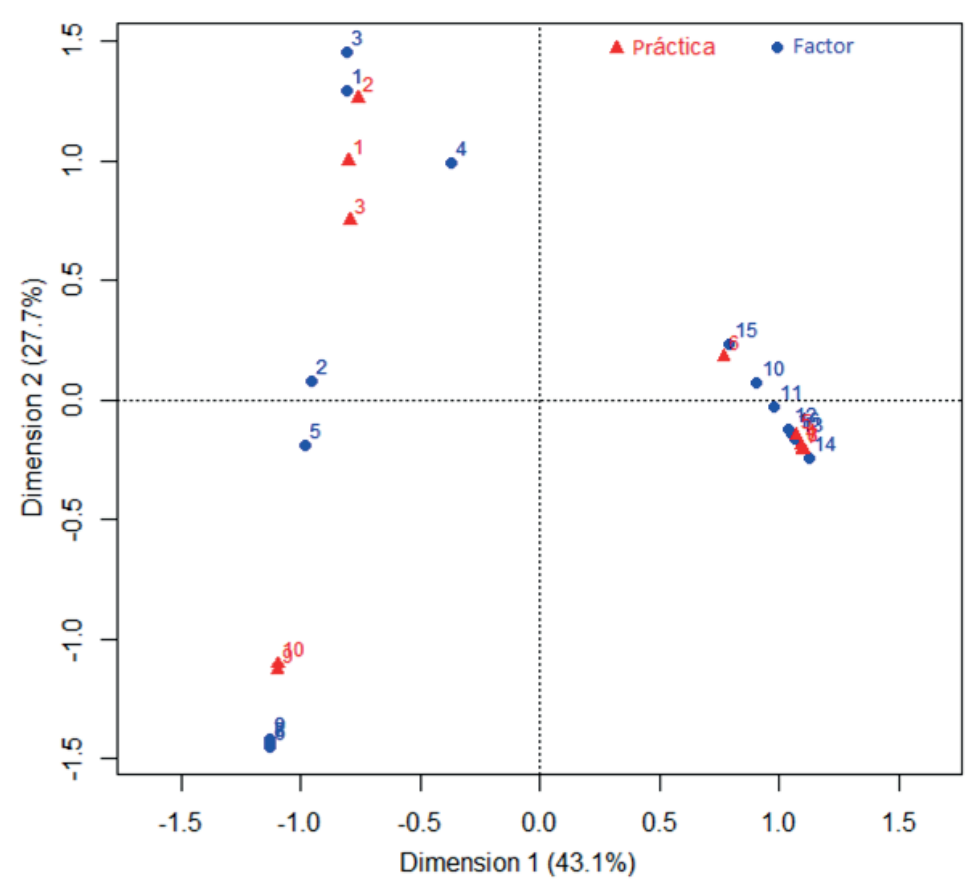

Figura 3. Análisis de correspondencia simple entre (Factores $\rightarrow$ Prácticas)

y P08, xiii) el factor F13 se relaciona con las prácticas P04, P06, P07 y P08, xiv) el factor F14 se relaciona con las prácticas P04, P05, P07 y P08, xv) el factor F15 se relaciona con la práctica P06, xvi) el factor F16 se relaciona con las prácticas P04, P05, P06, P07 y P08.

\subsection{Análisis de correspondencia Múltiple (ACM)}

Con este análisis se estudiará cómo se relacionan los factores con cada práctica y qué tanto contribuyen, a partir de los gráficos ilustrados. 
5.4.1 Influencia del factor $(F 01 \rightarrow P 01, P 02$, P03): La figura 4 muestra la distribución de los datos según las calificaciones, sobre la percepción de los encuestados acerca del factor F01, sobre las prácticas P01, P02, P03. Se observa que el nivel de influencia del factor se divide en dos grupos, un grupo de calificación 4 y 5 y el otro grupo de calificación solo "3: Medio".

En la tabla 8, se observa que la percepción de los encuestados, acerca de la influencia del factor F01 sobre las prácticas $\mathrm{P} 01, \mathrm{P} 02, \mathrm{P} 03$, la calificación ha sido "Muy Alta" con el $63.3 \%$ del total de calificación, seguido de "Alta" con el $32.7 \%$, en promedio, las dos calificaciones más altas concentran el $96 \%$ de las calificaciones.

En la figura 5, se presenta sobre el primer plano factorial del ACM: las categorías de todos los factores (arriba-izquierda), los 50 encuestados (arriba-derecha), las categorías y los encuestados simultáneamente (abajo-izquierda) y la proyección del nivel de experiencia superpuesta con las categorías (abajo-derecha). Se pueden leer las principales asociaciones: Se encuentra que las categorías "5: Muy alto" y "4: Alto" de todas las prácticas (P01, P02, P03) están fuertemente asociadas al factor F01 (arriba-izquierda). Además, el nivel "1: Muy poco" está asociado a la práctica P02. Los encuestados indican que todas las prácticas están siendo influenciadas por el factor F01 (abajo-izquierda) y, por último, se observa que todos niveles de tiempo de experiencia indican que están asociados a los niveles mayores e iguales a "3: Medio" de influencia del factor sobre las prácticas (abajo-derecha). En forma similar, se realiza el análisis de los otros factores con las prácticas, cuyo detalle se encuentra en [79].

5.4.2 Otras Relaciones: En la tabla 9, se puede observar las demás relaciones ACM obtenidas. De igual manera, se puede advertir acerca de la relación (influencia) entre el factor F02 sobre las prácticas (P01, P02, P03, P09, P10), la calificación ha sido "Alta" con el 52.8 $\%$, seguido de "Muy Alta" con el $26 \%$, en promedio las dos calificaciones concentran el $78.8 \%$ de las calificaciones (ver columna 4+5), similarmente se pude observar la influencia de los otros factores sobre las prácticas.

Observe además que los factores que presentan mayores calificaciones promedios entre "Muy Alta y Alta" son H16.6 ("Clara estrategia de TI, principios y políticas" $\rightarrow$ "Planeamiento estratégico de TI") y H1.1 ("Adecuado involucramiento de TI por parte de la alta dirección" $\rightarrow$ "Comité directivo") con valores promedio al $100 \%$ y $98 \%$, respectivamente. También, las hipótesis con menores calificaciones son H11.10, H10.10 y H15.6, con valores que no sobrepasan el promedio del $50 \%$ de las calificaciones entre "Muy Alta" y "Alta".

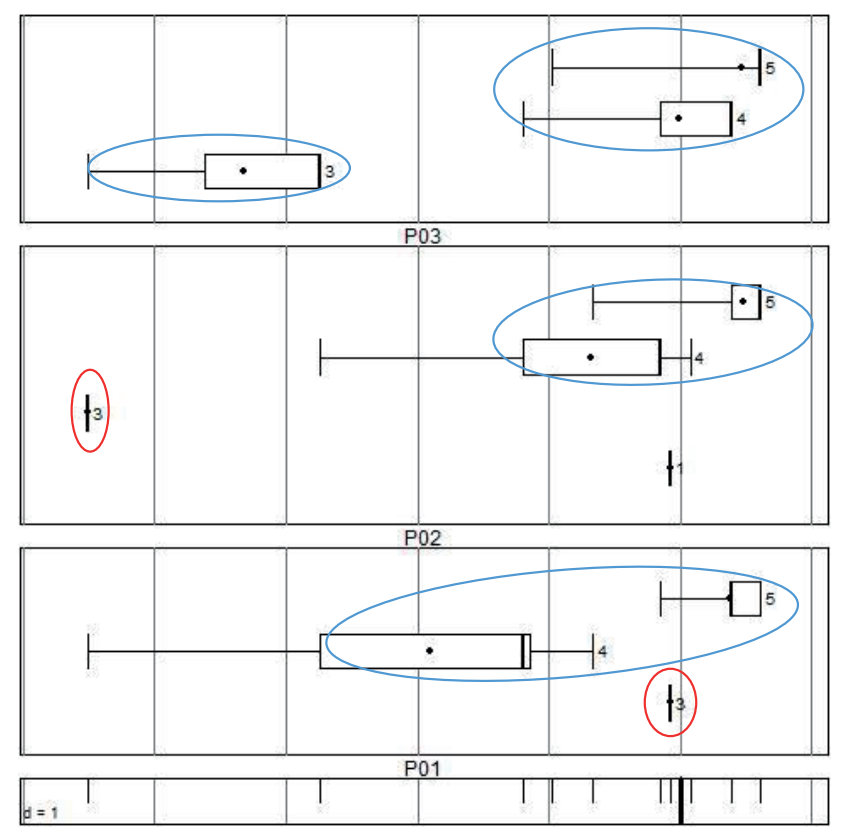

Figura 4. Distribución de calificaciones de la influencia $(F 01 \rightarrow$ P01, P02, P03)

Tabla 8. Calificación de encuestados sobre el nivel de influencia del factor F01 sobre P01, P02, P03

\begin{tabular}{ccccccc}
\hline \multicolumn{7}{c}{ Calificación } \\
\hline Relación & $\mathbf{1}$ & $\mathbf{2}$ & $\mathbf{3}$ & $\mathbf{4}$ & $\mathbf{5}$ & $\mathbf{( 4 + 5 )}$ \\
$\mathrm{H} 1.1$ & 0 & 0 & 0.02 & 0.16 & 0.82 & 98 \\
$\mathrm{H} 1.2$ & 0.02 & 0 & 0.02 & 0.32 & 0.64 & 96 \\
$\mathrm{H} 1.3$ & 0 & 0 & 0.06 & 0.5 & 0.44 & 94 \\
\hline Promedio $(4+5)$ & & & & & & 96 \\
\hline
\end{tabular}




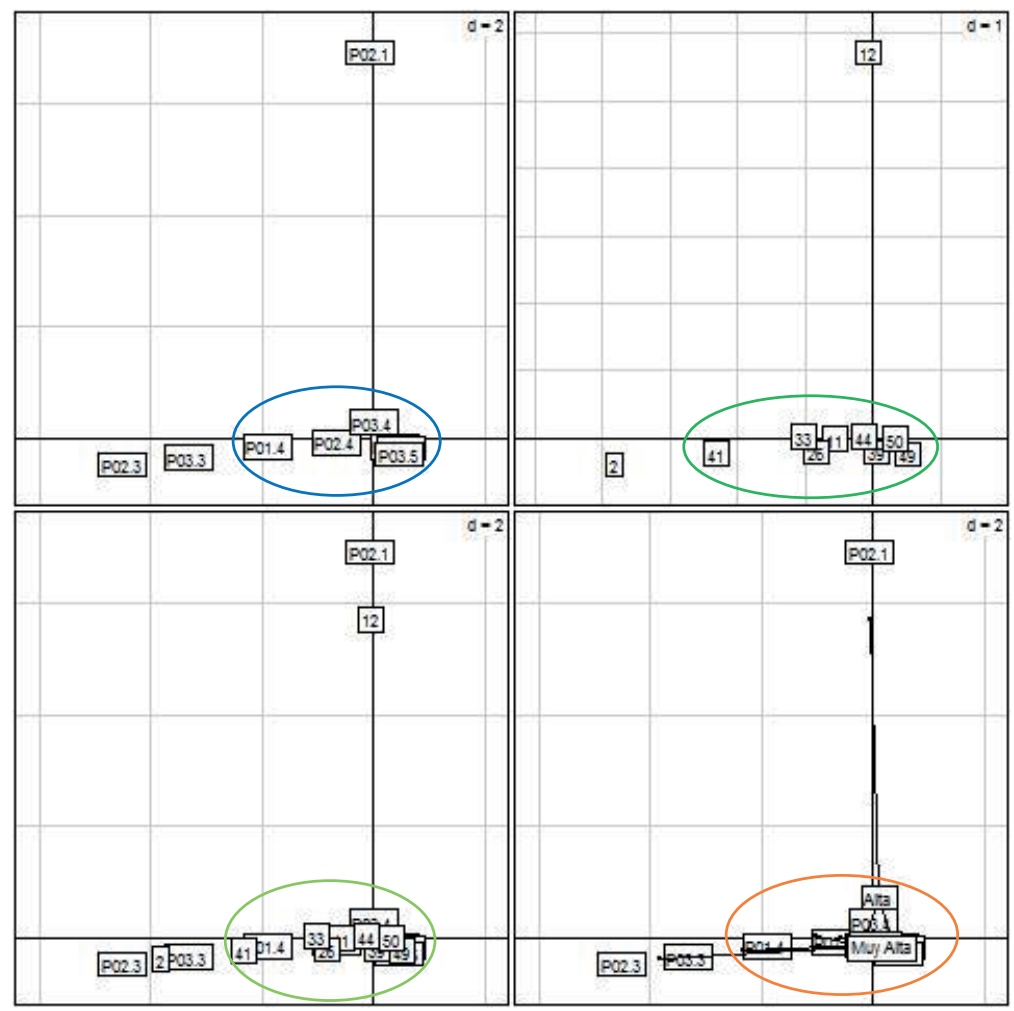

Figura 5. Plano factorial del ACM (F01 $\rightarrow$ P01, P02, P03)

Tabla 9. Calificación de la influencia (factores $\rightarrow$ prácticas)

\begin{tabular}{|c|c|c|c|c|c|c|c|}
\hline \multirow{2}{*}{ ACM } & \multirow{2}{*}{ Hipótesis } & \multicolumn{6}{|c|}{ Calificación } \\
\hline & & 1 & 2 & 3 & 4 & 5 & $(4+5) \%$ \\
\hline \multirow{6}{*}{$\mathrm{F} 02 \rightarrow \mathrm{P} 01, \mathrm{P} 02, \mathrm{P} 03, \mathrm{P} 09, \mathrm{P} 10$} & $\mathrm{H} 2.1$ & 0 & 0.02 & 0.28 & 0.44 & 0.26 & 70 \\
\hline & $\mathrm{H} 2.2$ & 0 & 0.02 & 0.28 & 0.44 & 0.26 & 70 \\
\hline & $\mathrm{H} 2.3$ & 0.02 & 0.04 & 0.14 & 0.5 & 0.3 & 80 \\
\hline & $\mathrm{H} 2.9$ & 0 & 0.02 & 0.14 & 0.62 & 0.22 & 84 \\
\hline & $\mathrm{H} 2.10$ & 0 & 0 & 0.1 & 0.64 & 0.26 & 90 \\
\hline & \multicolumn{6}{|c|}{ Promedio $(4+5)$} & 78 \\
\hline \multirow{4}{*}{$\mathrm{F03} \rightarrow \mathrm{P01}, \mathrm{P02}$} & H3.1 & 0 & 0.02 & 0.16 & 0.52 & 0.3 & 82 \\
\hline & H3.2 & 0.02 & 0.02 & 0.1 & 0.52 & 0.34 & 86 \\
\hline & \multicolumn{6}{|c|}{ Promedio $(4+5)$} & 84 \\
\hline & H4.1 & 0 & 0 & 0.18 & 0.62 & 0.2 & 82 \\
\hline \multirow{4}{*}{$\mathrm{F} 04 \rightarrow \mathrm{P} 01, \mathrm{P} 02, \mathrm{P} 03, \mathrm{P} 06$} & $\mathrm{H} 4.2$ & 0.02 & 0.02 & 0.14 & 0.52 & 0.3 & 82 \\
\hline & $\mathrm{H} 4.3$ & 0 & 0 & 0.12 & 0.58 & 0.3 & 88 \\
\hline & $\mathrm{H} 4.6$ & 0 & 0 & 0.04 & 0.66 & 0.3 & 96 \\
\hline & \multicolumn{6}{|c|}{ Promedio $(4+5)$} & 87 \\
\hline \multirow{5}{*}{$\mathrm{F} 05 \rightarrow \mathrm{P} 01, \mathrm{P} 03, \mathrm{P} 09, \mathrm{P} 10$} & H5.1 & 0 & 0.02 & 0.2 & 0.5 & 0.28 & 78 \\
\hline & $\mathrm{H} 5.3$ & 0 & 0 & 0.22 & 0.58 & 0.2 & 78 \\
\hline & H5.9 & 0 & 0 & 0.18 & 0.54 & 0.28 & 82 \\
\hline & $\mathrm{H} 5.10$ & 0 & 0 & 0.18 & 0.52 & 0.3 & 82 \\
\hline & \multicolumn{6}{|c|}{ Promedio $(4+5)$} & 80 \\
\hline $\mathrm{F} 06 \rightarrow \mathrm{P} 09$ & H6.9 & 0 & 0 & 0.1 & 0.4 & 0.5 & 90 \\
\hline \multirow{3}{*}{$\mathrm{F} 07 \rightarrow \mathrm{P} 09, \mathrm{P} 10$} & $\mathrm{H} 7.9$ & 0 & 0.02 & 0.14 & 0.6 & 0.24 & 84 \\
\hline & $\mathrm{H} 7.10$ & 0 & 0.02 & 0.14 & 0.5 & 0.34 & 84 \\
\hline & \multicolumn{6}{|c|}{ Promedio $(4+5)$} & 84 \\
\hline \multirow[t]{3}{*}{$\mathrm{F} 08 \rightarrow \mathrm{P} 09, \mathrm{P} 10$} & H8.9 & 0.02 & 0.02 & 0.16 & 0.56 & 0.24 & 80 \\
\hline & $\mathrm{H} 8.10$ & 0 & 0.02 & 0.16 & 0.64 & 0.18 & 82 \\
\hline & \multicolumn{6}{|c|}{ Promedio $(4+5)$} & 81 \\
\hline
\end{tabular}




\begin{tabular}{|c|c|c|c|c|c|c|c|}
\hline \multirow{2}{*}{$\mathrm{ACM}$} & \multirow{2}{*}{ Hipótesis } & \multicolumn{6}{|c|}{ Calificación } \\
\hline & & 1 & 2 & 3 & 4 & 5 & $(4+5) \%$ \\
\hline $\mathrm{F} 09 \rightarrow \mathrm{P} 10$ & $\mathrm{H} 9.10$ & 0 & 0 & 0.12 & 0.46 & 0.42 & 88 \\
\hline \multirow{3}{*}{$\mathrm{F} 10 \rightarrow \mathrm{P} 05, \mathrm{P} 06$} & $\mathrm{H} 10.5$ & 0.04 & 0.12 & 0.4 & 0.38 & 0.06 & 44 \\
\hline & H10.6 & 0 & 0.06 & 0.2 & 0.5 & 0.24 & 74 \\
\hline & \multicolumn{6}{|c|}{ Promedio $(4+5)$} & 59 \\
\hline \multirow{4}{*}{$\mathrm{F} 11 \rightarrow \mathrm{P} 04, \mathrm{P} 05, \mathrm{P} 06$} & H11.4 & 0.02 & 0.06 & 0.4 & 0.46 & 0.06 & 52 \\
\hline & $\mathrm{H} 11.5$ & 0.02 & 0.18 & 0.32 & 0.36 & 0.12 & 48 \\
\hline & H11.6 & 0 & 0.06 & 0.22 & 0.48 & 0.24 & 72 \\
\hline & \multicolumn{6}{|c|}{ Promedio (4+5) } & 57.3 \\
\hline \multirow{4}{*}{$\mathrm{F} 12 \rightarrow \mathrm{P} 05, \mathrm{P} 06, \mathrm{P} 07, \mathrm{P} 08$} & $\mathrm{H} 12.6$ & 0 & 0 & 0.08 & 0.52 & 0.4 & 92 \\
\hline & $\mathrm{H} 12.7$ & 0 & 0.02 & 0.08 & 0.56 & 0.34 & 90 \\
\hline & $\mathrm{H} 12.8$ & 0 & 0.02 & 0.04 & 0.56 & 0.38 & 94 \\
\hline & $\mathrm{H} 12.10$ & 0 & 0.02 & 0.1 & 0.56 & 0.32 & 88 \\
\hline \multicolumn{8}{|c|}{ Promedio (4+5) } \\
\hline \multirow{5}{*}{$\mathrm{F} 13 \rightarrow \mathrm{P} 04, \mathrm{P} 05, \mathrm{P} 06, \mathrm{P} 07, \mathrm{P} 08$} & H13.6 & 0 & 0.08 & 0.38 & 0.42 & 0.12 & 54 \\
\hline & $\mathrm{H} 13.7$ & 0 & 0.04 & 0.3 & 0.56 & 0.1 & 66 \\
\hline & H13.8 & 0 & 0 & 0.32 & 0.56 & 0.12 & 68 \\
\hline & H13.9 & 0 & 0 & 0.38 & 0.54 & 0.08 & 62 \\
\hline & H13.10 & 0 & 0.1 & 0.32 & 0.44 & 0.14 & 58 \\
\hline \multirow{6}{*}{$\mathrm{F} 14 \rightarrow \mathrm{P04}, \mathrm{P05}, \mathrm{P07}, \mathrm{P08}$} & \multicolumn{6}{|c|}{ Promedio $(4+5)$} & 58 \\
\hline & $\mathrm{H} 14.4$ & 0.02 & 0.02 & 0.34 & 0.46 & 0.16 & 62 \\
\hline & $\mathrm{H} 14.5$ & 0 & 0.16 & 0.3 & 0.44 & 0.1 & 54 \\
\hline & $\mathrm{H} 14.7$ & 0.02 & 0.04 & 0.28 & 0.54 & 0.12 & 66 \\
\hline & $\mathrm{H} 14.8$ & 0 & 0.06 & 0.32 & 0.38 & 0.24 & 62 \\
\hline & \multicolumn{6}{|c|}{ Promedio $(4+5)$} & 61 \\
\hline $\mathrm{F} 15 \rightarrow \mathrm{P} 06$ & H15.6 & 0.02 & 0.12 & 0.44 & 0.4 & 0.02 & 42 \\
\hline \multirow[t]{6}{*}{$\mathrm{F} 16 \rightarrow \mathrm{P} 04, \mathrm{P} 05, \mathrm{P} 06, \mathrm{P} 07, \mathrm{P} 08$} & H16.6 & 0 & 0 & 0 & 0.48 & 0.52 & 100 \\
\hline & $\mathrm{H} 16.7$ & 0 & 0 & 0.08 & 0.62 & 0.3 & 92 \\
\hline & H16.8 & 0 & 0 & 0.08 & 0.62 & 0.3 & 92 \\
\hline & H16.9 & 0 & 0.02 & 0.08 & 0.66 & 0.24 & 90 \\
\hline & H16.10 & 0 & 0 & 0.04 & 0.7 & 0.26 & 96 \\
\hline & \multicolumn{6}{|c|}{ Promedio $(4+5)$} & 94 \\
\hline
\end{tabular}

\subsection{Prueba de Hipótesis}

En esta sección, se aplica la distribución t-Student, con la finalidad de constatar las hipótesis planteadas. Se formularon la hipótesis nula $(\mathrm{Ho})$ y la hipótesis alternativa (Ha), y se definieron las siguientes reglas de decisión:

- Ho: $\mu<3.5$ (Los encuestados opinan que el grado de relación entre la práctica y el factor tiene una media menor a 3.5).

- Ha: $\mu>=3.5$ (Los encuestados opinan que el grado de relación entre la práctica y el factor tiene una media mayor o igual que 3.5).

Se ha considerado $\mu>=3.5$ porque las calificaciones 4 y 5 corresponden a "Alta" y "Muy Alta", respectivamente, y los valores mayores o iguales a 3.5 se interpretan mejor como "Alta" que "Media".

El valor del nivel de significancia $(\alpha)$ para este estudio es 0.05 . Si la probabilidad de error (valor $\mathrm{p}$ ) es mayor que el nivel de significancia $(\alpha)$, se acepta la hipótesis nula $H o$ y, por lo tanto, se rechaza la hipótesis alternativa $\mathrm{Ha}$. Si la probabilidad de error (valor p) es menor que el nivel de significancia $(\alpha)$, la hipótesis nula Ho se rechaza y, por lo tanto, se acepta la hipótesis alternativa $\mathrm{Ha}$.

Como se muestra en las hipótesis (ver tabla 10) (H1.1, H1.2, H1.3, H2.1, H2.2, H2.3, H2.9, H2.10, H3.1, H3.2, H4.1, H4.2, H4.3, H4.6, H5.1, H5.3, H5.9, H5.10, H6.9, H7.9, H7.10, H8.9, H8.10, H9.10, H10.6, H11.6, H12.5, H12.6, H12.7, H12.8, H13.4, H13.7, H13.8, H14.4, H14.7, H14.8, H16.4, H16.5, H16.6, H16.7, H16.8), el valor del error de probabilidad ( $\mathrm{p}$-value) es menor que $0.05(\mathrm{p}<0.05)$, por lo que se toma la hipótesis alternativa, y (Ha) es aceptada con un nivel de confianza de $95 \%$, que quiere decir que los factores tienen "alta influencia" sobre las prácticas de alineamiento de TI. También se observa que las hipótesis (H10.5, H11.4, H11.5, H13.5, H13.6, H14.5, H15.6), no han sido soportadas, ya que el error de probabilidad ( $p$-value) es mayor que 0.05 ( $p>0.05$ ), al $95 \%$ de confianza, lo que quiere decir que la relación entre los factores $\rightarrow$ prácticas: $(\mathrm{F} 10 \rightarrow \mathrm{P} 05),(\mathrm{F} 11 \rightarrow \mathrm{P} 04),(\mathrm{F} 11 \rightarrow$ $\mathrm{P} 05),(\mathrm{F} 13 \rightarrow \mathrm{P} 05),(\mathrm{F} 14 \rightarrow \mathrm{P} 05),(\mathrm{F} 15 \rightarrow \mathrm{P} 06)$ tienen solo "influencia media". 
La prueba de hipótesis en la tabla 10 confirma 41 relaciones de 48 hipótesis planteadas, siendo las hipótesis rechazadas las relaciones H10.5, H11.5, H13.5, H14.5, H11.4, H13.6, H15.6.
Las influencias de los factores "ambiente regulatorio y requerimiento de cumplimiento" (F10), "suficiente soporte financiero" (F11), "complejidad de TI" (F13), "dificultad de la entrega del valor y beneficios" (F14),

Tabla 10. Resumen de la prueba de hipótesis t-Student

\begin{tabular}{|c|c|c|c|c|c|c|c|c|}
\hline \multirow{2}{*}{ № } & \multirow{2}{*}{ Hipótesis } & \multirow{2}{*}{$\mathbf{t}$} & \multirow{2}{*}{ Df } & \multirow{2}{*}{ pvalue } & \multirow{2}{*}{ Confianza \% } & \multicolumn{2}{|c|}{ Media Estimada } & \multirow{2}{*}{ Soportado } \\
\hline & & & & & & Min & Max & \\
\hline 1 & $\mathrm{H} 1.1$ & 20,348 & 49 & 0.0000 & 95 & $4,671,613$ & $4,928,387$ & $\mathrm{SI}$ \\
\hline 2 & $\mathrm{H} 1.2$ & 10,227 & 49 & 0.0000 & 95 & $4,351,712$ & $4,768,288$ & SI \\
\hline 3 & $\mathrm{H} 1.3$ & 10,330 & 49 & 0.0000 & 95 & $4,208,807$ & $4,551,193$ & SI \\
\hline 4 & $\mathrm{H} 2.1$ & 3,923 & 49 & 0.0001 & 95 & $3,714,608$ & $4,165,392$ & $\mathrm{SI}$ \\
\hline 5 & $\mathrm{H} 2.2$ & 4,123 & 49 & 0.0001 & 95 & $3,766,521$ & $4,273,479$ & $\mathrm{SI}$ \\
\hline 6 & $\mathrm{H} 2.3$ & 5,709 & 49 & 0.0000 & 95 & $3,849,918$ & $4,230,082$ & SI \\
\hline 7 & $\mathrm{H} 2.9$ & 7,989 & 49 & 0.0000 & 95 & $3,993,988$ & $4,326,012$ & SI \\
\hline 8 & $\mathrm{H} 2.10$ & 10,193 & 49 & 0.0000 & 95 & $4,110,166$ & $4,409,834$ & $\mathrm{SI}$ \\
\hline 9 & H3.1 & 5,769 & 49 & 0.0000 & 95 & $3,891,001$ & $4,308,999$ & SI \\
\hline 10 & H3.2 & 5,431 & 49 & 0.0000 & 95 & $3,903,196$ & $4,376,804$ & SI \\
\hline 11 & H4.1 & 5,908 & 49 & 0.0000 & 95 & $3,843,124$ & $4,196,876$ & SI \\
\hline 12 & $\mathrm{H} 4.2$ & 4,697 & 49 & 0.0000 & 95 & $3,820,428$ & $4,299,572$ & SI \\
\hline 13 & $\mathrm{H} 4.3$ & 7,646 & 49 & 0.0000 & 95 & $4,001,270$ & $4,358,730$ & SI \\
\hline 14 & $\mathrm{H} 4.6$ & 10,193 & 49 & 0.0000 & 95 & $4,110,166$ & $4,409,834$ & SI \\
\hline 15 & H5.1 & 5,058 & 49 & 0.0000 & 95 & $3,825,474$ & $4,254,526$ & SI \\
\hline 16 & H5.10 & 6,360 & 49 & 0.0000 & 95 & $3,924,110$ & $4,315,890$ & SI \\
\hline 17 & H5.3 & 5,187 & 49 & 0.0000 & 95 & $3,794,038$ & $4,165,962$ & SI \\
\hline 18 & H5.9 & 6,261 & 49 & 0.0000 & 95 & $3,907,419$ & $4,292,581$ & $\mathrm{SI}$ \\
\hline 19 & H6.9 & 9,498 & 49 & 0.0000 & 95 & $4,209,571$ & $4,590,429$ & SI \\
\hline 20 & H7.10 & 6,320 & 49 & 0.0000 & 95 & $3,950,135$ & $4,369,865$ & SI \\
\hline 21 & H7.9 & 5,802 & 49 & 0.0000 & 95 & $3,866,055$ & $4,253,945$ & $\mathrm{SI}$ \\
\hline 22 & $\mathrm{H} 8.10$ & 5,187 & 49 & 0.0000 & 95 & $3,794,038$ & $4,165,962$ & SI \\
\hline 23 & H8.9 & 4,137 & 49 & 0.0001 & 95 & $3,746,844$ & $4,213,156$ & $\mathrm{SI}$ \\
\hline 24 & H9.10 & 8,348 & 49 & 0.0000 & 95 & $4,107,419$ & $4,492,581$ & $\mathrm{SI}$ \\
\hline 25 & $\mathrm{H} 10.5$ & $-1,556$ & 49 & 0.9369 & 95 & $3,041,626$ & $3,558,374$ & NO \\
\hline 26 & H10.6 & 3,582 & 49 & 0.0004 & 95 & $3,684,383$ & $4,155,617$ & SI \\
\hline 27 & $\mathrm{H} 11.4$ & -0.179 & 49 & 0.5708 & 95 & $3,255,855$ & $3,704,145$ & NO \\
\hline 28 & $\mathrm{H} 11.5$ & -0.859 & 49 & 0.8028 & 95 & $3,099,363$ & $3,660,637$ & NO \\
\hline 29 & H11.6 & 3,371 & 49 & 0.0007 & 95 & $3,661,532$ & $4,138,468$ & SI \\
\hline 30 & $\mathrm{H} 12.5$ & 6,961 & 49 & 0.0000 & 95 & $3,983,690$ & $4,376,310$ & $\mathrm{SI}$ \\
\hline 31 & $\mathrm{H} 12.6$ & 9,341 & 49 & 0.0000 & 95 & $4,143,590$ & $4,496,410$ & SI \\
\hline 32 & $\mathrm{H} 12.7$ & 7,500 & 49 & 0.0000 & 95 & $4,027,077$ & $4,412,923$ & SI \\
\hline 33 & $\mathrm{H} 12.8$ & 8,746 & 49 & 0.0000 & 95 & $4,116,178$ & $4,483,822$ & SI \\
\hline 34 & $\mathrm{H} 13.4$ & 2,302 & 49 & 0.0128 & 95 & $3,525,375$ & $3,874,625$ & $\mathrm{SI}$ \\
\hline 35 & $\mathrm{H} 13.5$ & 0.993 & 49 & 0.1628 & 95 & $3,377,148$ & $3,862,852$ & NO \\
\hline 36 & $\mathrm{H} 13.6$ & 0.698 & 49 & 0.2442 & 95 & $3,349,689$ & $3,810,311$ & NO \\
\hline 37 & H13.7 & 2,219 & 49 & 0.0156 & 95 & $3,520,772$ & $3,919,228$ & $\mathrm{SI}$ \\
\hline 38 & $\mathrm{H} 13.8$ & 3,320 & 49 & 0.0009 & 95 & $3,618,433$ & $3,981,567$ & $\mathrm{SI}$ \\
\hline 39 & $\mathrm{H} 14.4$ & 1,865 & 49 & 0.0341 & 95 & $3,482,988$ & $3,957,012$ & SI \\
\hline 40 & H14.5 & -0.160 & 49 & 0.5631 & 95 & $3,228,152$ & $3,731,848$ & NO \\
\hline 41 & H14.7 & 1,736 & 49 & 0.0444 & 95 & $3,468,547$ & $3,931,453$ & $\mathrm{SI}$ \\
\hline 42 & $\mathrm{H} 14.8$ & 2,409 & 49 & 0.0099 & 95 & $3,549,728$ & $4,050,272$ & SI \\
\hline 43 & H15.6 & $-1,985$ & 49 & 0.9736 & 95 & $3,057,331$ & $3,502,669$ & NO \\
\hline 44 & $\mathrm{H} 16.4$ & 6,989 & 49 & 0.0000 & 95 & $3,941,731$ & $4,298,269$ & SI \\
\hline 45 & H16.5 & 10,048 & 49 & 0.0000 & 95 & $4,076,000$ & $4,364,000$ & SI \\
\hline 46 & H16.6 & 14,291 & 49 & 0.0000 & 95 & $4,376,574$ & $4,663,426$ & $\mathrm{SI}$ \\
\hline 47 & $\mathrm{H} 16.7$ & 8,752 & 49 & 0.0000 & 95 & $4,054,685$ & $4,385,315$ & SI \\
\hline 48 & $\mathrm{H} 16.8$ & 8,752 & 49 & 0.0000 & 95 & $4,054,685$ & $4,385,315$ & SI \\
\hline
\end{tabular}


sobre la práctica "Metodología de gestión de proyectos" (P05), no fue soportada probablemente debido a que la mayoría de los encuestados pertenecen a las entidades públicas más importantes del país, que cuenta con bastos recursos financieros y personal especializado, este último no considera como una obligación el ambiente regulatorio y los requerimientos de cumplimiento, sino más bien como una presión mimético [10], y conocen los beneficios de implementar la gestión de proyecto, por todo ello estos factores poco influyen en P05.

El factor "Suficiente soporte financiero" (F11) no afecta a la "medición de desempeño" (P04), debido a que esta práctica es usual y política de TI en estas instituciones, y los encuestados no perciben el soporte financiero detrás de ellos.

Los factores "Complejidad de TI" (F13) y "Soporte externo de proveedores y consultorías" (F15) poco afectan a la práctica "Planeamiento estratégico de TI" (P06), debido a que las instituciones encuestadas poseen personal técnico especializado y el presupuesto adecuado que permite atender la complejidad de las TI y en general no requiere de soporte de proveedores externos.

\section{Conclusiones.}

Se han identificado 16 de los 52 factores para implementar prácticas de GTI que afectan a la implemen- tación de prácticas ATI en instituciones públicas. Del mismo modo, de la literatura se ha identificado 30 prácticas de GTI, donde 10 de estas están orientadas al ATI para el sector público.

A diferencia de trabajos anteriores que han identificado factores que afectan a la implementación de GTI o factores para el alineamiento, estos no indican a qué prácticas en particular afectan. Por esta razón, en este trabajo se ha identificado 48 relaciones de factores que afectan a las prácticas de ATI. Los resultados del estudio aplicados a 50 responsables de las oficinas de informática del sector público peruano, empleando el análisis de correspondencia simple (AC) y el análisis de correspondencia múltiple (ACM), muestra que 41 de las 48 de las relaciones propuestas han tenido una calificación entre "Alta" y "Muy Alta".

Finalmente, a través de la prueba de t-student se corroboró 41 relaciones sobre 15 factores que influyen en 10 prácticas ATI.

\section{Agradecimiento}

Los autores agradecen a todas las gerencias de informática de las instituciones del poder ejecutivo y de organismos autónomos de Perú que han participado en el presente estudio.

\section{APÉNDICES}

Tabla A. Prácticas de ATI

\begin{tabular}{|c|c|c|}
\hline ID & Prácticas & Fuente \\
\hline 1 & Comité Estratégico de $\mathrm{TI}$ a nivel de la junta directiva & {$[15,16,20,22,80]$} \\
\hline 2 & Comité de Dirección de TI (Evaluación de inversión de TI / priorización a nivel ejecutivo y alta dirección) & {$[5,15,20,38,22,40,56]$} \\
\hline 3 & $\mathrm{ClO}$ en el comité Ejecutivo & {$[15,16,20,37,7,56]$} \\
\hline 4 & $\mathrm{CIO}$ reportando al CEO & {$[15,40,56]$} \\
\hline 5 & Comité directivo de proyectos de TI & {$[15,8]$} \\
\hline 6 & Comité de auditoría a nivel de la junta directiva & [15] \\
\hline 7 & Roles y responsabilidades en el gobierno de $\mathrm{TI}$ & {$[20,21]$} \\
\hline 8 & Comité directivo de seguridad de TI & {$[17,7]$} \\
\hline 9 & Comité directivo de Arquitectura & {$[17,8]$} \\
\hline 10 & Integración de tareas de (alineamiento/gobierno) en roles y responsabilidades & {$[17,7,56,20,21,76]$} \\
\hline 11 & Planeamiento estratégico de TI & {$[17,16,44,20,21,76]$} \\
\hline 12 & Sistema de Medición del desempeño (ejemplo: IT balanced scorecard) & {$[15,8,74,16,38,22,80,81]$} \\
\hline 13 & Gestión del Portafolio de Proyectos de TI & {$[17,8,21,19]$} \\
\hline 14 & Costeo de servicios de TI - costo total de propiedad (actividad basada en costeo) & {$[17,56]$} \\
\hline 15 & Acuerdo de Nivel de Servicio - SLA & {$[73,56,14]$} \\
\hline 16 & Framework de Gobierno de TI (Ejemplo COBIT, ISO 38500) & {$[73,44,14]$} \\
\hline 17 & Uso de una metodología de Gestión de Proyectos de TI & {$[73,40,44,14,39,21,81]$} \\
\hline 18 & Control del presupuesto de Tl y presentación de informes & {$[15,71,14,8]$} \\
\hline 19 & La gestión de los beneficios y presentación de informes & {$[15,14]$} \\
\hline 20 & Asegurar la optimización de Riesgo & {$[8,14,44]$} \\
\hline 21 & Gestión de Inversión de TI (proceso de selección de inversión) & {$[71,8,14,57,44,5,21,81]$} \\
\hline 22 & Aseguramiento de gobierno de TI y autoevaluación & {$[15,8]$} \\
\hline
\end{tabular}




\begin{tabular}{lll}
\hline ID & Prácticas & Fuente \\
\hline 23 & Asegurar la optimización de Recursos. & {$[14,44]$} \\
\hline 24 & Rotación de trabajo y entrenamiento multifuncional entre (TI/negocio) & {$[16,20,37,73]$} \\
25 & Gestión del Conocimiento (en Gobierno de TI) & {$[73]$} \\
26 & Sistemas de Comunicación Interna corporativa. & {$[38,22,22,37]$} \\
27 & Campañas de concienciación de gobierno de TI & {$[25,17]$} \\
28 & Ética o cultura de cumplimiento & {$[38,74]$} \\
29 & Asegurar Transparencia para los interesados & {$[14]$} \\
30 & Reuniones informales entre negocios y ejecutivos de TI/ Gestión Senior & {$[17,73,16]$} \\
\hline
\end{tabular}

Tabla B. Factores que influyen para implementar PATI

\begin{tabular}{|c|c|c|}
\hline ID & Factores & Fuente \\
\hline 1 & Adecuado apoyo de la gestión y responsabilidad & {$[24]$} \\
\hline 2 & Alta gerencia menos comprometida & {$[38,75]$} \\
\hline 3 & Falta de apoyo y compromiso de la alta dirección & [30] \\
\hline 4 & Involucrar y conseguir el apoyo de la alta dirección & {$[11,22]$} \\
\hline 5 & soporte de la alta dirección & {$[27,62]$} \\
\hline 6 & Ambiente Regulatorio y requerimiento de cumplimiento & {$[24]$} \\
\hline 7 & Complejidad de marcos de ITG existentes & {$[51]$} \\
\hline 8 & Cumplimiento de regulación y mitigación de riesgos & {$[28]$} \\
\hline 9 & presión externa de gobierno y la industria & {$[27]$} \\
\hline 10 & Falta de comunicación (entre TI y Negocios) & {$[29,30]$} \\
\hline 11 & Falta de comunicación persuasiva & {$[76]$} \\
\hline 12 & Falta estrecha relación entre IT/Business & {$[57]$} \\
\hline 13 & Falta de beneficios percibidos & [51] \\
\hline 14 & Adecuado Soporte Financiero & {$[24]$} \\
\hline 15 & Costo/financiamiento/presupuesto & {$[30]$} \\
\hline 16 & Soporte de recurso financiero y humano & {$[28]$} \\
\hline 17 & Inadecuado soporte de recursos financieros & [29] \\
\hline 18 & Limitación de presupuesto & [57] \\
\hline 19 & adecuado capacidades de TI y del equipo & {$[24]$,} \\
\hline 20 & Adecuado entrenamiento en $\mathrm{TI}$ & {$[27]$} \\
\hline 21 & Falta de capacidades y conocimiento en TI & [33] \\
\hline 22 & Proveer concientización de GTI y entrenamiento para el uso óptimo de TI. & {$[17]$} \\
\hline 23 & Clara estrategia de $\mathrm{Tl}$, principios y políticas & {$[11,24,45]$} \\
\hline 24 & $\begin{array}{l}\text { Consolidar, comunica y aplique políticas y pautas para la adquisición, implementación y uso rentable de TI en toda la } \\
\text { organización }\end{array}$ & [11] \\
\hline 25 & Falta de principios y políticas claras de gobierno de $\mathrm{Tl}$ & [29] \\
\hline 26 & Falta de Claros principios de GTI & [57] \\
\hline 27 & Falta de estrecha relación entre TI y negocios & [57] \\
\hline 28 & Falta de principios y políticas de TI para el gobierno de TI & [29] \\
\hline 29 & El personal de TI carece de orientación empresarial & {$[15]$} \\
\hline 30 & No hay perspectiva de integración de negocios y TI & {$[82]$} \\
\hline 31 & Adecuado involucramiento de los Stakeholders & {$[24]$} \\
\hline 32 & Falta de involucramiento de los stakeholders interno y externos & [30] \\
\hline 33 & Inadecuado involucramiento de los Stakeholder & [29] \\
\hline 34 & Resistencia al Cambio & {$[30,33]$} \\
\hline 35 & Resistencia para aceptar estándares y Políticas & [57] \\
\hline 36 & Soporte Externo & {$[83]$} \\
\hline 37 & Soporte externo de proveedores y consultores & {$[27,31]$} \\
\hline 38 & Cultura organizacional & {$[24,55,46]$} \\
\hline
\end{tabular}




\begin{tabular}{lll}
\hline ID & Factores & Fuente \\
\hline 39 & Cultura organizacional y Políticas & {$[30]$} \\
\hline 40 & Buena relación entre personal de TI y negocios & {$[58,56,42]$} \\
\hline 41 & Competencia Organizacional de TI & {$[53]$} \\
\hline 42 & Madurez & {$[55]$} \\
\hline 43 & Complejidad & {$[31,33]$} \\
\hline 44 & Dificultad en demostrar valor y beneficios & {$[30,31]$} \\
\hline 45 & Disponibilidad al cambio & {$[77,72]$} \\
\hline 46 & Disponibilidad de Expertise Interna de TI & {$[27]$} \\
\hline 47 & Conocimiento de dominio compartido entre personal de Tl y negocios & {$[64,84],[16,50]$} \\
\hline 48 & Estructura de gobierno corporativo & {$[53]$} \\
49 & Estructura de la Organización & {$[54]$} \\
50 & Influencia organizacional & {$[52]$} \\
\hline 51 & Liderazgo de TI & {$[11,42,56]$} \\
\hline 52 & Política Organizacional & {$[33,40]$} \\
\hline
\end{tabular}

Tabla C. Promedio, varianza y moda de la calificacion de los encuestados (Factores $\rightarrow$ Prácticas)

\begin{tabular}{|c|c|c|c|c|}
\hline $\mathbf{N}^{\circ}$ & Hipótesis & Promedio & Desv. Estándar & Moda \\
\hline 1 & $\mathrm{H} 1.1$ & 4,8 & 0,45 & 5 \\
\hline 2 & $\mathrm{H} 1.2$ & 4,56 & 0,73 & 5 \\
\hline 3 & $\mathrm{H} 1.3$ & 4,38 & 0,6 & 4 \\
\hline 4 & $\mathrm{H} 2.1$ & 3,94 & 0,79 & 4 \\
\hline 5 & $\mathrm{H} 2.2$ & 4,02 & 0,89 & 4 \\
\hline 6 & $\mathrm{H} 2.3$ & 4,04 & 0,67 & 4 \\
\hline 7 & $\mathrm{H} 2.9$ & 4,16 & 0,58 & 4 \\
\hline 8 & $\mathrm{H} 2.10$ & 4,26 & 0,53 & 4 \\
\hline 9 & H3.1 & 4,1 & 0,74 & 4 \\
\hline 10 & H3.2 & 4,14 & 0,83 & 4 \\
\hline 11 & H4.1 & 4,02 & 0,62 & 4 \\
\hline 12 & $\mathrm{H} 4.2$ & 4,06 & 0,84 & 4 \\
\hline 13 & $\mathrm{H} 4.3$ & 4,18 & 0,63 & 4 \\
\hline 14 & $\mathrm{H} 4.6$ & 4,26 & 0,53 & 4 \\
\hline 15 & H5.1 & 4,04 & 0,75 & 4 \\
\hline 16 & H5.3 & 3,98 & 0,65 & 4 \\
\hline 17 & H5.9 & 4,1 & 0,68 & 4 \\
\hline 18 & H5.10 & 4,12 & 0,69 & 4 \\
\hline 19 & H6.9 & 4,4 & 0,67 & 5 \\
\hline 20 & $\mathrm{H} 7.9$ & 4,06 & 0,68 & 4 \\
\hline 21 & $\mathrm{H} 7.10$ & 4,16 & 0,74 & 4 \\
\hline 22 & H8.9 & 3,98 & 0,82 & 4 \\
\hline 23 & $\mathrm{H} 8.10$ & 3,98 & 0,65 & 4 \\
\hline 24 & H9.10 & 4,3 & 0,68 & 4 \\
\hline 25 & $\mathrm{H} 10.5$ & 3,3 & 0,91 & 3 \\
\hline 26 & H10.6 & 3,92 & 0,83 & 4 \\
\hline 27 & $\mathrm{H} 11.4$ & 3,48 & 0,79 & 4 \\
\hline 28 & $\mathrm{H} 11.5$ & 3,38 & 0,99 & 4 \\
\hline 29 & H11.6 & 3,9 & 0,84 & 4 \\
\hline 30 & $\mathrm{H} 12.5$ & 4,18 & 0,69 & 4 \\
\hline 31 & $\mathrm{H} 12.6$ & 4,32 & 0,62 & 4 \\
\hline 32 & $\mathrm{H} 12.7$ & 4,22 & 0,68 & 4 \\
\hline
\end{tabular}




\begin{tabular}{llccc}
\hline $\mathbf{N}^{\circ}$ & Hipótesis & Promedio & Desv. Estándar & Moda \\
\hline $\mathbf{3 3}$ & H12.8 & 4,3 & 0,65 & 4 \\
$\mathbf{3 4}$ & H13.4 & 3,7 & 0,61 & 4 \\
\hline $\mathbf{3 5}$ & H13.5 & 3,62 & 0,85 & 4 \\
\hline $\mathbf{3 6}$ & H13.6 & 3,58 & 0,81 & 4 \\
$\mathbf{3 7}$ & H13.7 & 3,72 & 0,7 & 4 \\
\hline $\mathbf{3 8}$ & H13.8 & 3,8 & 0,64 & 4 \\
\hline $\mathbf{3 9}$ & H14.4 & 3,72 & 0,83 & 4 \\
\hline $\mathbf{4 0}$ & H14.5 & 3,48 & 0,89 & 4 \\
\hline $\mathbf{4 1}$ & H14.7 & 3,7 & 0,81 & 4 \\
\hline $\mathbf{4 2}$ & H14.8 & 3,8 & 0,88 & 4 \\
\hline $\mathbf{4 3}$ & H15.6 & 3,28 & 0,78 & 3 \\
\hline $\mathbf{4 4}$ & H16.4 & 4,12 & 0,63 & 4 \\
\hline $\mathbf{4 5}$ & H16.5 & 4,22 & 0,51 & 4 \\
\hline $\mathbf{4 6}$ & H16.6 & 4,52 & 0,5 & 5 \\
\hline $\mathbf{4 7}$ & H16.7 & 4,22 & 0,58 & 4 \\
\hline $\mathbf{4 8}$ & H16.8 & 4,22 & 0,58 & 4 \\
\hline
\end{tabular}

\section{Referencias}

[1] West, D. M. (2004). Egovernment and the transformation of service delivery and citizen attitudes. Public administration review, 64(1), 15-27.

[2] Orozco, J., Tarhini, A., Masa'deh, R. (Moh'd T., \& Tarhini, T. (2015). A Framework of IS/Business Alignment Management Practices to Improve the Design of IT Governance Architectures. International Journal of Business and Management, 10(4), 1-12. https://doi.org/10.5539/ijbm.v10n4p1

[3] Orozco, J., Tarhini, A., Masa'deh, R. (Moh'd T., \& Tarhini, T. (2015). A Framework of IS/Business Alignment Management Practices to Improve the Design of IT Governance Architectures. International Journal of Business and Management, 10(4), 1-12.

[4] Kappelman, L., McLean, E., Johnson, V., \& Torres, R. (2016). The 2015 SIM IT Issues and Trends Study. MIS Quarterly Executive, 15(1).

[5] Winkler, T. J. (2013). IT Governance Mechanisms and Administration / IT Alignment in the Public Sector: A Conceptual Model and Case Validation IT Governance. In Wirtschaftsinformatik (p. 53)

[6] Xue, Y., Liang, H. \& Boulton, W. R. (2006). Information Technology Governance in Processes: The impact of Investment Characteristics, External Enviroment, and Internal Context. Management Information System Quarterly, 32(1), 67-96

[7] ITGI (2003) Board Briefing on IT Governance

[8] ITGI (2008). IT Governance Global Status Report 2008. In Control.

[9] Vander Elst, S., \& De Rynck, F. (2014). Alignment processes in public organizations: an interpretive approach. Information Polity, 19(3, 4), 195-206.

[10] Pereira, G. V., Luciano, E. M., Macadar, M. A., \& Daniel, V. M. (2013). Information technology governance practices adoption through an institutional perspective: the perception of
Brazilian and American CIOs. In 2013 46th Hawaii International Conference on System Sciences (pp. 4446-4455). IEEE.

[11] Nfuka, E. N., \& Rusu, L. (2010). Critical Success Factors for Effective IT Governance in the Public Sector Organizations in a Developing Country: The Case of Tanzania. In ECIS (p. 128).

[12] Henderson, J. C., \& Venkatraman, H. (1999). Strategic alignment: Leveraging information technology for transforming organizations. IBM systems journal, 38(2.3), 472-484.

[13] Khadem, K. N. (2007). Aligning enterprise and information technology strategy: A study of the correlation between strategic alignment and adaptation of enterprise-wide strategy formulation processes (Doctoral dissertation, Capella University).

[14] ISACA (2012). A Business Framework For The Governance And Management Of Enterprise IT.

[15] De Haes, S., \& van Grembergen, W. (2009). An Exploratory Study into IT Governance Implementations and its Impact on Business/IT Alignment. Information Systems Management, 26(2), 123-137.

[16] Peterson, R. (2004). Crafting information technology governance. Information Systems Management, 21(4), 7-22.

[17] De Haes, S., \& Van Grembergen, W. (2008). Practices in IT governance and business/IT alignment. Proceedings of the Annual Hawaii International Conference on System Sciences, 2(June 2016), 1-6.

[18] Schlosser, F. (2011). IT Governance Practices For Improving Strategic And Operational Business-IT Alignment. In PACIS (p. 167).

[19] Tambotoh, J. J. C., Kosala, R., Ranti, B., Isa, S. M., \& Pudjianto, B. W. (2017). A Conceptual Model for Creating Effective Public Value Through Key Practices in Information Technology Governance Mechanisms. (November), 128-132.

[20] Qassimi, N. Al, \& Rusu, L. (2015). IT Governance in a Public Organization in a Developing Country: A Case Study of 
a Governmental Organization. Procedia Computer Science, 64, 450-456.

[21] Satidularn, C., Tanner, K., \& Wilkin, C. (2011). Exploring IT Governance Arrangements In Practice: The Case Of A Utility Organisation In Thailand. In PACIS (P 163).

[22] Maidin, S. S., \& Arshad, N. H. (2010a). Information technology governance practices in Malaysian public sector. 2010 International Conference on Financial Theory and Engineering, ICFTE 2010, 281-285.

[23] Leidecker, J. K., \& Bruno, A. V. (1984). Identifying and using critical success factors. Long range planning, 17(1), 23-32.

[24] Alreemy, Z., Chang, V., Walters, R., \& Wills, G. (2016). Critical success factors (CSFs) for information technology governance (ITG). International Journal of Information Management, 36(6), 907-916

[25] Nfuka, E. N., \& Rusu, L. (2013). Critical success framework for implementing effective IT governance in Tanzanian public sector organizations. Journal of Global Information Technology Management, 16(3), 53-77.

[26] Aggarwal, H. (2010). Critical Success Factors in IT Alignment in Public Sector Petroleum Industry of India. International Journal of Innovation, Management and Technology, 1(1), 56-63.

[27] Aoun, C., Vatanasakdakul, S., \& Chen, Y. (2011). IT governance framework adoption: Establishing success factors. In IFIP International Working Conference on Governance and Sustainability in Information Systems-Managing the Transfer and Diffusion of IT (pp. 239-248).

[28] Urbach, N., Buchwald, A., \& Ahlemann, F. (2013). Understanding IT Governance Success And Its Impact: Results From An Interview Study. In ECIS (p. 55).

[29] Lee, J., Lee, C., \& Kap-Young, J. (2008). Governance inhibitors in it strategy and management: An empirical study of korean enterprises. Global Economic Review, 37(1), 1-22.

[30] Othman, M. F. I., Chan, T., \& Foo, E. (2011). IT Governance Adoption in Malaysia: A Preliminary Investigation. Australasian Conference on Information Systems (ACIS 2011), Paper 69.

[31] Othman, M. F. I., Pee, N. C., Rahim, Y. A., Sulaiman, H. A., Othman, M. A., \& Aziz, M. Z. A. A. (2018). Using Analytical Hierarchy Process (AHP) to Evaluate Barriers in Adopting Formal IT Governance Practices. Journal of Telecommunication, Electronic and Computer Engineering (JTEC), 10(1-6), $35-40$.

[32] Fink, K. and Ploder, C. (2008), "Decision support framework for the implementation of IT governance", Proceedings of the 41st Hawaii International Conference on System Sciences, Waikoloa, Big Island, $\mathrm{HI}$.

[33] Othman, M. F. I., \& Chan, T. (2013). Barriers to formal IT Governance practice - Insights from a qualitative study. Proceedings of the Annual Hawaii International Conference on System Sciences, 4415-4424.

[34] Kurti, I., Barolli, E., \& Sevrani, K. (2014). Effective IT Governance in the Albanian Public Sector - A Critical Success Factors Approach. The Electronic Journal of Information Systems in Developing Countries, 63(1), 1-22.

[35] Schlosser, F., Wagner, H. T., \& Coltman, T. (2012). Reconsidering the dimensions of business-IT alignment. In 2012 45th
Hawaii International Conference on System Sciences (pp. 5053-5061). IEEE.

[36] Pereira, R., Almeida, R., \& Mira, M. (2014). IT Governance Patterns in the Portuguese Financial Industry. In 2014 47th Hawaii International Conference on System Sciences (pp. 4386-4395). IEEE.

[37] Nfuka, E. N., Rusu, L., Johannesson, P., \& Mutagahywa, B. (2009). The state of IT governance in organizations from the public sector in a developing country. In 2009 42nd Hawaii International Conference on System Sciences (pp. 1-12). IEEE.

[38] Ali, S., \& Green, P. (2012). Effective information technology (IT) governance mechanisms: An IT outsourcing perspective. Information Systems Frontiers, 14(2), 179-193.

[39] Adaba, G. B., \& Rusu, L. (2014). IT Governance Practices in a Public Organization in Ghana. International Journal of Innovation in the Digital Economy, 5(2), 14-23.

[40] Wilkin, C. L., \& Riddett, J. (2009). IT governance challenges in a large not-for-profit healthcare organization: The role of intranets. Electronic Commerce Research, 9(4), 351-374.

[41] Weill, P., \& Ross, J. W. (2005). It Governance on One Page. In SSRN.

[42] Luftman, J., Papp, R., \& Brier, T. (1999). Enablers and inhibitors of business-IT alignment. Communications of the Association for information Systems, 1(1), 11.

[43] Wilkin, C. L., \& Riddett, J. L. (2008). Issues for IT governance in a large not-for-profit organization: A case study. In 2008 International MCETECH Conference on e-Technologies (mcetech 2008) (pp. 193-202).

[44] ITGI, I. (2007). COBIT 4.1. Framework Control Objective Management Guidelines Maturity Model.

[45] International Organization for Standardization (ISO), ISO/IEC 38500:2008, Corporate Governance of information technology, Switzerland, 2008; www.iso.org

[46] Janssen, L. A., Luciano, E. M., \& Testa, M. G. (2013). The influence of organizational culture on IT governance: Perception of a group of IT managers from Latin American companies. In 2013 46th Hawaii International Conference on System Sciences (pp. 4485-4494).

[47] Abu-Musa, A.A (2007). Exploring information technology governance (ITG) in developing countries: an empirical study. The International Journal of Digital Accounting Research, 7(13), 71-117.

[48] Pérez Lorences, P., \& García Ávila, L. F. (2013). The evaluation and improvement of IT governance. Journal of Information Systems and Technology Management, 10(2), 219-234.

[49] Spafford, G. (2003). The benefits of standard IT governance frameworks. IT management april, 2003 (December 2000)

[50] Peterson, R. R. (2001). Information governance: an empirical investigation into the differentiation and integration of strategic decision-making for IT. The Netherlands: Tilburg University.

[51] Vogt, M., Küller, P., Hertweck, D., \& Hales, K. (2011). Adapting IT Governance Frameworks using Domain Specific Requirements Methods: Examples from Small \& Medium Enterprises and Emergency Management. In AMCIS.

[52] Chiu, Y. H., Liu, L., \& Chi, Y. P. (2011). Study on correlation between critical successful factors of IT governance and go- 
vernance performance. Journal of Convergence Information Technology, 6(5).

[53] Chin, P. O., Brown, G. A., \& Hu, Q. (2004). The impact of mergers and acquisitions on IT governance structures: A case study. Journal of Global Information Management, 12(4), 50-74.

[54] Cochran, M. (2010, January). Proposal of an operations department model to provide IT governance in organizations that don't have IT C-level executives. In 2010 43rd Hawaii International Conference on System Sciences (pp. 1-10). IEEE.

[55] Pereira, R., \& da Silva, M. M. (2012). IT governance implementation: The determinant factors. Communications of the IBIMA, 2012, 1.

[56] Weill, P., \& Ross, J. W. (2004). IT governance: How top performers manage IT decision rights for superior results. Harvard Business Press.

[57] Jairak, K., Praneetpolgrang, P., (2011, November). A holistic survey of IT governance in the Thai university through IT executive perspectives. In international Conference on Informatics Engineering and Information Science (pp. 435-4471). Springer, Berlin, Heidelberg.

[58] Teo, T. S., \& Ang, J. S. (1999). Critical success factors in the alignment of IS plans with business plans. International Journal of Information Management, 19(2), 173-185.

[59] Luftman, J. N., \& Rajkumar, K. (2007). "Alignment. A line” has been drawn”, MIS Quarterly Executive, 6(3).

[60] Wibowo, A. M., \& Yuwono, B. (2008). Driving Factors. Enablers \& Inhibitors of IT Value Delivery \& Risk Management in IT Governance.

[61] ISACA, I. (2011). Global Status Report on the Governance of Enterprise IT (GEIT)-2011.

[62] Vatanasakdakul, S., Aoun \& Chen, Y. (2017). Chassing Succes: An Emprirical for IT governance frameworks adoption in Australia. Science, Technology and Society, 22(2),182-221.

[63] ITGI \& PwC (2009). An executive view of IT governance.

[64] Reich, B. H., \& Benbasat, I. (2000). Factors that influence the social dimension of alignment between business and information technology objectives. MIS quarterly, 81-113.

[65] Cohen, W. M., \& Levinthal, D. A. (1990). Absorptive capacity: A new perspective on learning and innovation. Administrative science quarterly, 35(1), 128-152

[66] Nelson, K. M., \& Cooprider, J. G. (1996). The contribution of shared knowledge to IS group performance. MIS quarterly, 409-432.

[67] Silva, L., \& Figueroa B, E. (2002). Institutional intervention and the expansion of ICTs in Latin America: The case of Chile. Information Technology \& People, 15(1), 8-25.

[68] Streiner, D. L. (2003). Being inconsistent about consistency: When coefficient alpha does and doesn't matter. Journal of personality assessment, 80(3), 217-222.

[69] Ojo, A., Janowski, T., \& Shareef, M. (2009). Aligning electronic government and public administration reform programs-process, tool and case study. In Proceedings of the 9th European Conference on eGovernment (pp. 510-521).
[70] Chaudhuri, A. (2011). Enabling effective IT governance: Leveraging ISO/IEC 38500: 2008 and COBIT to achieve business-IT alignment. Edpacs, 44(2), 1-18

[71] Luftman, J., Ben-Zvi, T., Dwivedi, R., \& Rigoni, E. H. (2010). IT Governance: An alignment maturity perspective. International Journal of IT/Business Alignment and Governance (IJITBAG), 1(2), 13-25.

[72] Luftman, J. (2000). Assessing Business-IT Alignment Maturity. Communications of the AIS.

[73] De Haes, S., \& Van Grembergen, W. (2006). IT Governance best practices in Belgian organisations. In Proceedings of the 39th Annual Hawaii International Conference on System Sciences (HICSS'06)(Vol. 8, pp. 195b-195b). IEEE.

[74] Maidin, S. S., \& Arshad, N. H. (2010b). IT governance practices model in IT project approval and implementation in Malaysian public sector. ICEIE 2010 - 2010 International Conference on Electronics and Information Engineering, Proceedings, 1 (Iceie 2010), 532-536.

[75] Ferguson, C., Green, P., Vaswani, R., \& Wu, G. (2013). Determinants of effective information technology governance. International Journal of Auditing, 17(1), 75-99.

[76] De Souza Bermejo, P. H., Tonelli, A. O., Zambalde, A. L., dos Santos, P. A., \& Zuppo, L. (2014). Evaluating IT governance practices and business and IT outcomes: A quantitative exploratory study in Brazilian companies. Procedia Technology, 16, 849-857.

[77] Luftman, J., \& Kempaiah, R. (2007). An Update on Business-IT Alignment:" A Line" Has Been Drawn. MIS Quarterly Executive, 6(3).

[78] Webb, P., Pollard, C., \& Ridley, G. (2006, January). Attempting to define IT governance: Wisdom or folly?. In Proceedings of the 39th Annual Hawaii International Conference on System Sciences (HICSS'06) (Vol. 8, pp. 194a-194a). IEEE.

[79] Juan Diaz, Factores para implementar prácticas para el alineamiento estratégico de TI en el sector público: Un enfoque desde el gobierno de TI, Mg. Tesis, UNMSM, (2019), Impress.

[80] Ali, S., \& Green, P. (2007). IT Governance Mechanisms in Public Sector Organisations: An Australian Context. Journal of Global Information Management (JGIM), 15(4), 41-63.

[81] Wiedenhoft, G. C., Luciano, E. M., \& Magnagnagno, O. A. (2017). Information Technology Governance In Public Organizations: identifying mechanisms that meet its goals while respecting principles. JISTEM-Journal of Information Systems and Technology Management, 14(1), 69-87.

[82] Altemimi, M. A. H., \& Zakaria, M. S. (2015, December). Developing factors for effective IT governance mechanism. In 2015 9th Malaysian Software Engineering Conference (MySEC)(pp. 245-251). IEEE..

[83] Letsoalo, K., Brown, I., \& Njenga, K. N. (2006). An investigation of enablers and inhibitors of IT governance implementation: A case study of a South African enterprise. In Proceedings of the ITG International Conference (pp. 27-35).

[84] Chan, Y. E., \& Reich, B. H. (2007). IT alignment: what have we learned?. Journal of Information Technology, 22, 297315. 
